\title{
Nonlinear saturation of baroclinic instability: part II: continuously stratified fluid
}

Article

Published Version

Shepherd, T. G. (1989) Nonlinear saturation of baroclinic instability: part II: continuously stratified fluid. Journal of the Atmospheric Sciences, 46 (7). pp. 888-907. ISSN 1520-0469 doi: https://doi.org/10.1175/15200469(1989)046<0888:NSOBIP>2.0.CO;2 Available at https://centaur.reading.ac.uk/32905/

It is advisable to refer to the publisher's version if you intend to cite from the work. See Guidance on citing.

Published version at: http://dx.doi.org/10.1175/1520-0469(1989)046<0888:NSOBIP>2.0.CO;2

To link to this article DOI: http://dx.doi.org/10.1175/1520-

0469(1989)046<0888:NSOBIP>2.0.CO;2

Publisher: American Meteorological Society

All outputs in CentAUR are protected by Intellectual Property Rights law, including copyright law. Copyright and IPR is retained by the creators or other copyright holders. Terms and conditions for use of this material are defined in the End User Agreement.

www.reading.ac.uk/centaur 


\section{CentAUR}

Central Archive at the University of Reading

Reading's research outputs online 


\title{
Nonlinear Saturation of Baroclinic Instability. Part II: Continuously Stratified Fluid
}

\author{
THEODORE G. SHEPHERD \\ Department of Applied Mathematics and Theoretical Physics, University of Cambridge, Cambridge, U.K.
}

(Manuscript received 28 March 1988, in final form 15 September 1988)

\section{ABSTRACT}

Rigorous upper bounds are derived that limit the finite-amplitude growth of arbitrary nonzonal disturbances to an unstable baroclinic zonal flow in a continuously stratified, quasi-geostrophic, semi-infinite fluid. Bounds are obtained both on the depth-integrated eddy potential enstrophy and on the eddy available potential energy (APE) at the ground. The method used to derive the bounds is essentially analogous to that used in Part I of this study for the two-layer model: it relies on the existence of a nonlinear Liapunov (normed) stability theorem, which is a finite-amplitude generalization of the Charney-Stern theorem. As in Part 1, the bounds are valid both for conservative (unforced, inviscid) flow, as well as for forced-dissipative flow when the dissipation is proportional to the potential vorticity in the interior, and to the potential temperature at the ground.

The character of the results depends on the dimensionless external parameter $\gamma=f_{0}^{2} \xi / \beta_{0} N^{2} H$, where $\xi$ is the maximum vertical shear of the zonal wind, $H$ is the density scale height, and the other symbols have their usual meaning. When $\gamma \gg 1$, corresponding to "deep" unstable modes (vertical scale $\approx H$ ), the bound on the eddy potential enstrophy is just the total potential enstrophy in the system; but when $\gamma \ll 1$, corresponding to 'shallow' unstable modes ( vertical scale $\approx \gamma H$ ), the eddy potential enstrophy can be bounded well below the total amount available in the system. In neither case can the bound on the eddy APE prevent a complete neutralization of the surface temperature gradient, which is in accord with numerical experience.

For the special case of the Charney model of baroclinic instability, and in the limit of infinitesimal initial eddy disturbance amplitude, the bound states that the dimensionless eddy potential enstrophy cannot exceed $(\gamma+1)^{2} / 24 \gamma^{2} h$ when $\gamma \geqslant 1$, or $1 / 6 \gamma h$ when $\gamma \leqslant 1$; here $h=H N / f_{0} L$ is the dimensionless scale height, and $L$ is the width of the channel. These bounds are very similar to (though of course generally larger than) ad hoc estimates based on baroclinic-adjustment arguments.

The possibility of using these kinds of bounds for eddy-amplitude closure in a transient-eddy parameterization scheme is also discussed.

\section{Introduction}

In Part I of this study (Shepherd 1988a), rigorous upper bounds were derived on the finite-amplitude saturation of baroclinic instability in a two-layer quasigeostrophic fluid. These bounds restricted the growth of the potential enstrophy of the nonzonal part of the flow, and were obtained through the use of a Liapunov (normed) stability theorem which is the finite-amplitude generalization of the well-known small-amplitude Charney-Stern theorem. Comparison of the bounds with weakly nonlinear theory for the special case of the Phillips (1954) model of baroclinic instability showed very similar parameter dependences as well as remarkable quantitative agreement, suggesting that the saturation bounds might provide, inter alia, a useful way of determining eddy-amplitude closures in transient-eddy parameterization schemes. It is significant in this respect that the bounds are unconstrained by any assumptions about the nature of the initial non-

Corresponding author address: Dr. T. G. Shepherd, Department of Physics, University of Toronto, Toronto MSS IA7 Canada. zonal disturbance, and thus are not tied in any way to the concept of normal-mode instability.

Despite the utility of the two-layer model in elucidating fundamental dynamical processes, it nevertheless has serious shortcomings with regard to atmospheric applications. Not least is the fact that the truncated vertical modal representation introduces an unrealistic short-wave cutoff in the linear instability problem, as well as a minimum critical shear. Moreover, because the meridional temperature gradient is situated between the layers, any instability must be essentially internal, in the sense that the reversal of the potential-vorticity gradient must occur well within the interior of the atmosphere. This feature is certainly unrealistic as regards synoptic-scale baroclinic instability in our own atmosphere, where the potential-vorticity gradient is typically positive throughout the depth of the atmosphere and the instability is driven by the negative surface temperature gradient (e.g. Simmons and Hoskins 1976, 1978). Therefore, it seems appropriate to apply the methods of Part I to the more realistic problem of baroclinic instability in a continuously stratified fluid, including the Charney (1947) model as a special case. 
The plan of the paper is as follows. In section 2 the governing equations are reviewed, and the pseudomomentum (or generalized Eliassen-Palm) conservation law for finite-amplitude disturbances to zonal basic flows is established. This conservation law is then used to derive a generalized Charney-Stern stability theorem in section 3. In section 4, the methods of Part I are employed to deduce finite-amplitude saturation bounds for the Charney (1947) model of baroclinic instability; in this case the relevant Liapunov (normed) stability theorem is that given by (4.12) below. Bounds are derived both on the depth-integrated eddy potential enstrophy and on the eddy available potential energy (APE) at the ground. The bounds are compared to ad hoc baroclinic-adjustment estimates in section 5, and to Pedlosky's (1979b) weakly nonlinear theory in section 6 . Then in section 7 the problem of more general flow profiles is treated. Some implications of these ideas for transient-eddy parameterization are considered in section 8 , and the paper concludes with a discussion.

\section{Governing equations, and the pseudomomentum conservation law for disturbances to zonal flows}

The system under consideration in this paper is that of three-dimensional, baroclinic, quasi-geostrophic flow on a beta-plane, which is governed by conservation of quasi-geostrophic potential vorticity $P$ at each vertical level $z$ (where $z$ is a log-pressure vertical coordinate):

$$
\frac{D P}{D t}=0 \text {, }
$$

i.e.

$$
P_{t}+\partial(\Phi, P) \equiv P_{t}-\Phi_{y} P_{x}+\Phi_{x} P_{y}=0,
$$

where $\Phi$ is the geostrophic streamfunction, $J(\bullet, \bullet)$ the horizontal Jacobian operator, and

$P \equiv \Phi_{x x}+\Phi_{y y}+\frac{1}{\rho}\left\{\rho\left(f_{0} / N\right)^{2} \Phi_{z}\right\}_{z}+f_{0}+\beta_{0} y$.

Here $f=f_{0}+\beta_{0} y$ represents the beta-plane approximation to the Coriolis parameter, $\rho(z)$ is the (prescribed) reference-state density stratification, and $N(z)$ the reference-state buoyancy frequency (e.g., see Pedlosky $1979 \mathrm{a}, \S 6.5$ ). The derivations are performed assuming unforced, inviscid flow; but see the comments regarding forced-dissipative problems in section 9 . The domain is infinite in the zonal coordinate $x$ but bounded in the meridional coordinate $y$, with boundary conditions

$$
\Phi_{x}=0, \quad \bar{\Phi}_{y t}=0 \quad \text { at } \quad y=y_{1}, y_{2},
$$

where the overbar refers to a zonal average

$$
\bar{f} \equiv \lim _{X \rightarrow \infty} \frac{1}{2 X} \int_{-X}^{X} f(x) d x .
$$

The boundary conditions in the vertical are taken to be

$$
\frac{D}{D t} \Phi_{z}=0 \quad \text { at } \quad z=z_{1}, z_{2},
$$

with $z=z_{1}$ being the lower surface and $z=z_{2}$ the upper. (After section 3, attention will be restricted to the case of a semi-infinite domain; then one replaces the boundary condition at $z=z_{2}$ with a condition that the vertical component of the flux given in (2.13) vanish as $z \rightarrow \infty$, and all "upper boundary" effects vanish.) Conditions (2.4) are those derived under "Type 1" quasi-geostrophic scaling, under the assumption $f_{0}^{2} L^{2} /$ $g H \ll 1$ where $L$ is a characteristic length scale and $H$ the density scale height (see White 1977). While the assumption is suspect for planetary-scale motions, it is acceptable for synoptic-scale baroclinic instability (White and Gadian 1979) and thus (2.4) is appropriate here. The possibility of allowing the full boundary conditions, which include a term $N^{2} g^{-1} \partial \Phi / \partial t$ (where $g$ is the gravitational acceleration ), is considered in appen$\operatorname{dix}$ A.

The generalized Charney-Stern theorem derived in the next section arises from a finite-amplitude pseudomomentum conservation law, (2.18) below, which is obtained as follows. Consider a steady, $x$-invariant "basic state" $(\Phi, P)=(\Psi, Q)$, with a potential vorticity distribution

$Q(y, z)=\Psi_{y y}+\frac{1}{\rho}\left\{\rho\left(f_{0} / N\right)^{2} \Psi_{z}\right\}_{z}+f_{0}+\beta_{0} y$.

Defining the disturbance $(\psi, q)$ in the natural way

$$
\Phi \equiv \Psi+\psi, \quad P \equiv Q+q,
$$

so that

$$
q=\psi_{x x}+\psi_{y y}+\frac{1}{\rho}\left\{\rho\left(f_{0} / N\right)^{2} \psi_{z}\right\}_{z},
$$

and noting that the basic flow is itself an exact solution to (2.1) and (2.4), the disturbance potential vorticity is governed by the exact equation

$$
\frac{D q}{D t}=-\partial(\psi, Q)=-\psi_{x} Q_{y}=-\frac{D Q}{D t}
$$

for each $z$, with boundary conditions

$$
\begin{gathered}
\frac{D}{D t} \psi_{z}=-\partial\left(\psi, \Psi_{z}\right)=-\psi_{x} \Psi_{z y}=-\frac{D}{D t} \Psi_{z} \\
\text { at } z=z_{1}, z_{2}, \\
\psi_{x}=0, \quad \bar{\psi}_{y t}=0 \quad \text { at } y=y_{1}, y_{2} .
\end{gathered}
$$

The $x$-invariance of the basic state $Q(y, z)$ allows the definition of the inverse function $Y_{0}(Q ; z)$ in the 
interior of the fluid. Now introduce the second-order disturbance quantity

$A(Q, q ; z) \equiv-\rho \int_{0}^{q}\left\{Y_{0}(Q+\tilde{q} ; z)-Y_{0}(Q ; z)\right\} d \tilde{q} ;$

note that in the limit of small disturbance amplitude,

$$
A(Q, q ; z) \approx-\frac{\rho}{2} Y_{0}^{\prime}(Q ; z) q^{2}=-\frac{\rho}{2} \frac{q^{2}}{Q_{y}},
$$

which is just (the negative of) the linearized "EliassenPalm wave activity" (Andrews and McIntyre 1976; Held 1985). It may be verified from (2.11) that

$$
\begin{aligned}
& \frac{1}{\rho} \frac{\partial A}{\partial Q}=-Y_{0}(Q+q)+Y_{0}(Q)+q Y_{0}^{\prime}(Q) \\
& \frac{1}{\rho} \frac{\partial A}{\partial q}=-Y_{0}(Q+q)+Y_{0}(Q),
\end{aligned}
$$

and thence, using (2.8), that

$$
\begin{aligned}
\frac{D A}{D t}= & \rho q Y_{0}^{\prime}(Q) \psi_{x} Q_{y} \\
= & \rho\left(\psi_{x x}+\psi_{y y}\right) \psi_{x}+\left\{\rho\left(f_{0} / N\right)^{2} \psi_{z}\right\}_{z} \psi_{x} \\
= & \rho\left\{\frac{1}{2} \frac{\partial}{\partial x}\left(\psi_{x}^{2}-\psi_{y}^{2}\right)+\frac{\partial}{\partial y}\left(\psi_{x} \psi_{y}\right)\right\} \\
& +\frac{\partial}{\partial z}\left\{\rho\left(f_{0} / N\right)^{2} \psi_{x} \psi_{z}\right\}-\rho\left(f_{0} / N\right)^{2} \frac{\partial}{\partial x}\left(\frac{1}{2} \psi_{z}^{2}\right) \\
= & \frac{\partial}{\partial x}\left\{\frac{\rho}{2}\left(\psi_{x}^{2}-\psi_{y}{ }^{2}-\left(f_{0} / N\right)^{2} \psi_{z}^{2}\right)\right\} \\
& +\frac{\partial}{\partial y}\left\{\rho \psi_{x} \psi_{y}\right\}+\frac{\partial}{\partial z}\left\{\rho\left(f_{0} / N\right)^{2} \psi_{x} \psi_{z}\right\} .
\end{aligned}
$$

When averaged in $x$, this is just the quasi-geostrophic baroclinic version of the generalized Eliassen-Palm theorem of Killworth and McIntyre [1985, Eq. (5.17)]. Integration of the $x$-average of (2.13) across the channel and through the depth of the fluid then yields

$$
\begin{aligned}
\frac{d}{d t} \iint \bar{A} d y d z=\left.\int \rho\left(f_{0} / N\right)^{2} \overline{\psi_{x} \psi_{z}} d y\right|_{z=z_{2}} \\
-\left.\int \rho\left(f_{0} / N\right)^{2} \overline{\psi_{x} \psi_{z}} d y\right|_{z=z_{1}} .
\end{aligned}
$$

(It is the convention in this paper that, unless otherwise indicated, integrals over $y$ and $z$ refer to the full ranges $y_{1} \leqslant y \leqslant y_{2}$ and $z_{1} \leqslant z \leqslant z_{2}$.)

To obtain a conservation law from (2.14) it is necessary to again appeal to the $x$-invariance of the basic state, and define the function

$B(\theta, \theta)=-\frac{\rho f_{0} g}{N^{2} \Theta_{s}} \int_{0}^{\theta}\left\{Y_{0}(\Theta+\tilde{\theta})-Y_{0}(\theta)\right\} d \tilde{\theta}$.
Here $\theta(y, z)+\theta(x, y, z, t)$ is the deviation from the reference-state potential-temperature distribution $\Theta_{s}(z)$, divided into basic-state and disturbance components; recall that according to standard quasi-geostrophic scaling

$$
\theta=\left(\Theta_{s} f_{0} / g\right) \Psi_{z}, \quad \theta=\left(\Theta_{s} f_{0} / g\right) \psi_{z} .
$$

Using (2.9) and (2.16) it is readily verified that, analogously to the first equation of (2.13),

$$
\frac{D B}{D t}=\frac{\rho f_{0} g}{N^{2} \Theta_{s}} \theta \psi_{x}=\rho\left(f_{0} / N\right)^{2} \psi_{x} \psi_{z} .
$$

Substitution of (2.17) into (2.14) then yields the global conservation law

$$
\frac{d}{d t} J=0
$$

where

$$
\begin{aligned}
J \equiv & \iint \overline{A(Q, q ; z)} d y d z \\
& +\left.\int \overline{B(\Theta, \theta)} d y\right|_{z=z_{1}}-\left.\int \overline{B(\Theta, \theta)} d y\right|_{z=z_{2}} .
\end{aligned}
$$

is the integrated pseudomomentum. Equation (2.18) corresponds to the pseudoenergy conservation law (B7) of McIntyre and Shepherd (1987) for disturbances to nonparallel basic flows. The way in which the two kinds of conservation law are related to the symmetry properties of the basic state is discussed in section 7 of that paper.

\section{The generalized Charney-Stern theorem}

In this section the pseudomomentum conservation law (2.18) is used to derive a Liapunov stability theorem for disturbances to zonal flows. The theorem expresses nonlinear stability, in the sense that a disturbance norm at any time $t$ is bounded in terms of its value at $t=0$, and represents a finite-amplitude generalization of the well-known Charney-Stern stability theorem. The essential idea is originally due to Arnol'd (1966), and other applications of Arnol'd's method are described by Holm et al. (1985), Abarbanel et al. (1986), and McIntyre and Shepherd (1987).

The first point to note is that whenever the basicstate potential vorticity $Q$ is a monotonic function of $y$ at some level $z$, then $A(Q, q ; z)$ defined by $(2.11)$ is of definite sign for arbitrary disturbances $q$ at that level, and takes a sign opposite to that of $Q_{y}$. Similarly with $B(\Theta, \theta)$, defined by (2.15), which takes a sign opposite to that of $\theta_{y}$ whenever the latter is definite. Therefore, it may be seen from (2.19) that the pseudomomentum $J$ will be of definite sign whenever the sign of $Q_{y}$ throughout the domain is the same as that of $\Theta_{y}$ at $z$ $=z_{1}$, and the opposite to that of $\Theta_{y}$ at $z=z_{2}$. Since $J$ is constant in time, normal-mode instabilities are then 
precluded (because they would necessarily cause $|J|$ to increase): this is of course just the Charney-Stern theorem. But the stability is in fact nonlinear, as will now be demonstrated.

For definiteness, consider the case of $Q_{y}>0$ and suppose there exist constants $c, C, c_{1}, C_{1}$, and $c_{2}, C_{2}$ such that

$$
\begin{array}{lll}
0<c \leqslant Q_{y} \leqslant C<\infty & \text { for } & z \in\left(z_{1}, z_{2}\right), \\
0<c_{1} \leqslant \theta_{y} \leqslant C_{1}<\infty & \text { at } & z=z_{1}, \\
0<c_{2} \leqslant-\Theta_{y} \leqslant C_{2}<\infty & \text { at } & z=z_{2} .
\end{array}
$$

Now introduce the disturbance norm $\|\psi\|$ defined by

$$
\begin{array}{r}
\|\psi\|^{2} \equiv \iint \rho c^{-1} \overline{q^{2}} d y d z+\left.\int \frac{\rho f_{0} g}{N^{2} \theta_{s}} c_{1}^{-1} \overline{\theta^{2}} d y\right|_{z=z_{1}} \\
+\left.\int \frac{\rho f_{0} g}{N^{2} \Theta_{s}} c_{2}^{-1} \overline{\theta^{2}} d y\right|_{z=z_{2}}
\end{array}
$$

this evidently involves the potential enstrophy in the interior, plus the available potential energy on the upper and lower boundaries. Then noting the inequalities

$$
\begin{aligned}
& \frac{\rho q^{2}}{2 C}=\int_{0}^{q} \rho\left|Y_{0}^{\prime}(Q)\right|_{\min } \tilde{q} d \tilde{q} \leqslant|A(Q, q)| \\
& \quad \leqslant \int_{0}^{q} \rho\left|Y_{0}^{\prime}(Q)\right|_{\max } \tilde{q} d \tilde{q}=\frac{\rho q^{2}}{2 c}, \\
& \frac{\rho f_{0} g \theta^{2}}{2 N^{2} \Theta_{s} C_{i}}=\int_{0}^{\theta} \frac{\rho f_{0} g}{N^{2} \Theta_{s}}\left|Y_{0}^{\prime}(\theta)\right|_{\min } \tilde{\theta} d \tilde{\theta} \leqslant|B(\theta, \theta)| \\
& \quad \leqslant \int_{0}^{\theta} \frac{\rho f_{0} g}{N^{2} \Theta_{s}}\left|Y_{0}^{\prime}(\theta)\right|_{\max } \tilde{\theta} d \tilde{\theta}=\frac{\rho f_{0} g \theta^{2}}{2 N^{2} \Theta_{s} c_{i}},
\end{aligned}
$$

it follows that

$$
\begin{aligned}
& \frac{1}{2}\|\psi(t)\|^{2} \leqslant \frac{C}{c} \iint|\overline{A(t)}| d y d z \\
& +\left.\frac{C_{1}}{c_{1}} \int|\overline{B(t)}| d y\right|_{z=z_{1}} \\
& +\left.\frac{C_{2}}{c_{2}} \int|\overline{B(t)}| d y\right|_{z=z_{2}} \leqslant \max \left\{\frac{C}{c}, \frac{C_{1}}{c_{1}}, \frac{C_{2}}{c_{2}}\right\}|J(t)| \\
& =\max \left\{\frac{C}{c}, \frac{C_{1}}{c_{1}}, \frac{C_{2}}{c_{2}}\right\}|J(0)| \\
& \quad \leqslant \max \left\{\frac{C}{c}, \frac{C_{1}}{c_{1}}, \frac{C_{2}}{c_{2}}\right\} \frac{1}{2}\|\psi(0)\|^{2}
\end{aligned}
$$

which is a statement of Liapunov (normed) stability for the norm given by (3.2), under the conditions (3.1). It may be verified that (3.4) is equally valid for the norm obtained by replacing $c_{i}$ in (3.2) with $C_{i}$. Equation (3.4) is closely related to the pseudoenergy-based stability theorem discussed by McIntyre and Shepherd [1987, Eqs. (B8) and (B9)].

\section{Nonlinear saturation bounds for the Charney model}

In Part I of this study, the generalized Charney-Stern theorem was used to place rigorous upper bounds on the saturation of baroclinic instability in the two-layer model. The device used to obtain these bounds is briefly described as follows. A (possibly infinitesimal) nonzonal disturbance to an unstable zonal flow may be regarded as a finite-amplitude disturbance (including a zonal-mean part) to some stable "basic state"; the evolution in time of this latter disturbance is then constrained by the generalized (normed) Charney-Stern theorem. In particular, the nonzonal part of the disturbance (which is independent of the choice of the basic state) is thereby constrained, and by considering a family of basic states one may determine a best (i.e., least) upper bound for the family.

In the context of the two-layer model, there is a minimum critical shear and so it is natural to consider basic states that are similar in structure to the initial unstable zonal flow, but with subcritical shears. For a continuously stratified fluid, of course, minimum critical shears do not generally exist, and one must consider a slightly different strategy. To simplify matters, we focus in this section on the Charney (1947) model of baroclinic instability, leaving the consideration of more general profiles to section 7 .

The Charney model considers the instability of a zonal flow

$$
\hat{U}(z) \equiv-\frac{\partial \bar{\Phi}}{\partial y}=\xi z
$$

in the semi-infinite domain $0 \leqslant z<\infty$, and assumes an isothermal reference atmosphere so that

$$
\rho(z)=\rho_{0} e^{-z / H}
$$

with the scale height $H \equiv R T_{0} / g$ constant, and the buoyancy frequency $N$ likewise constant. It is convenient to nondimensionalize the equations with length scaled by the width of the channel $L \equiv y_{2}-y_{1}$, depth by $D \equiv f_{0} L / N$, velocity by $\xi D$, potential temperature by the constant reference temperature $T_{0}$, and density by $\rho_{0}$. In these units, the zonal-mean streamfunction corresponding to (4.1) is taken to be

$$
\bar{\Phi}(y, z)=-z(y-\lambda),
$$

with $\lambda$ an as yet arbitrary constant of integration, and the associated potential-vorticity distribution (2.2) is given by

$$
\begin{aligned}
\bar{P}(y, z) & \equiv \bar{\Phi}_{y y}+\bar{\Phi}_{z z}-h^{-1} \bar{\Phi}_{z}+f+\beta y \\
& =h^{-1}(y-\lambda)+f+\beta y,
\end{aligned}
$$

where

$$
h \equiv \frac{H N}{f_{0} L}, \quad \beta \equiv \frac{\beta_{0} L^{2}}{\xi D}=\frac{\beta_{0} N L}{f_{0} \xi}, \quad f \equiv \frac{f_{0} L}{\xi D}=\frac{N}{\xi} .
$$


It is clear from (4.4) that the meridional gradient of $\bar{P}$ is everywhere positive, while the temperature gradient at the ground,

$$
\frac{f_{0} \xi L}{g} \bar{\Phi}_{z y}=-\frac{f_{0} \xi L}{g},
$$

is everywhere negative; hence the conditions of the Charney-Stern theorem are decidedly not met, and indeed the flow is of course known to be unstable.

The problem of interest concerns the nonlinear development of an initial condition consisting of the zonal flow (4.3), (4.4), plus an arbitrary nonzonal disturbance. In particular, the goal is to determine some upper bound on the growth of the instability. As in Part $\mathrm{I}$, the strategy is to represent this initial condition as a disturbance $(\psi, q)$ to some stable basic state $(\Psi, Q)$, and then invoke the Charney-Stern theorem (3.4). A suitable basic state must have $\Psi_{z y}>0$ at the ground and $Q_{y}>0$ in the interior, and it is evident that it need differ from (4.3)-(4.5) only in the lowest part of the atmosphere. Consequently, we choose to consider basic flows of the form

$U(z)= \begin{cases}u_{0}-u_{1} z+u_{2}\left(h e^{z / h}-1\right), & \text { for } 0 \leqslant z \leqslant b \\ z, \text { for } z \geqslant b, & \end{cases}$

as sketched in Fig. 1. Imposing continuity of $U$ and $U_{z}$ at $z=b$ leads to the two constraints

$$
\begin{aligned}
u_{0}-u_{1} b+u_{2} h\left(e^{b / h}-1\right) & =b, \\
-u_{1}+u_{2} e^{b / h} & =1 .
\end{aligned}
$$

The potential vorticity distribution corresponding to (4.6) takes the simple form

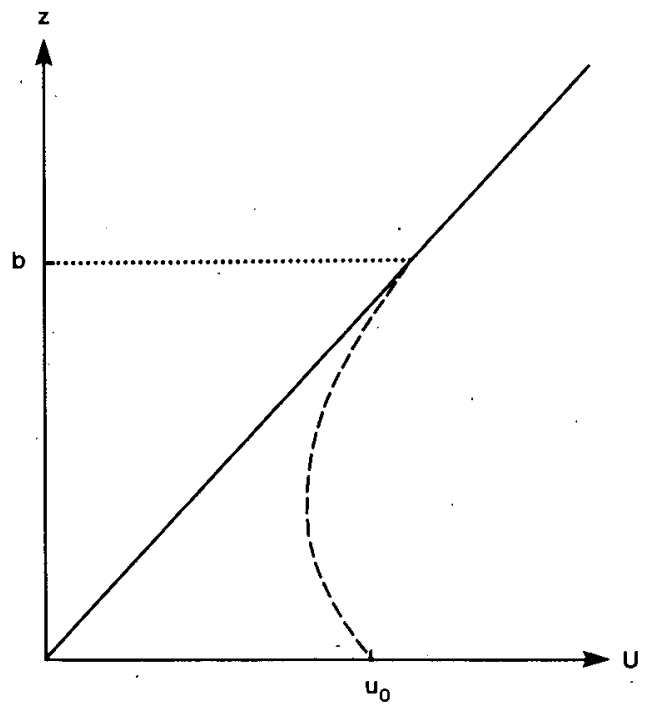

Fig. 1. Vertical profile of the unstable Charney flow (solid line) and the stable basic flow (4.6) (dashed line).

$$
\begin{aligned}
& Q(y, z) \\
& =\left\{\begin{array}{lll}
-h^{-1} u_{1}(y-\lambda)+f+\beta y, & \text { for } \quad 0 \leqslant z \leqslant b \\
h^{-1}(y-\lambda)+f+\beta y, & \text { for } \quad z \geqslant b,
\end{array}\right.
\end{aligned}
$$

which has a positive meridional gradient throughout the fluid provided that

$$
u_{1}<\beta h \text {; }
$$

while the surface temperature distribution associated with (4.6) is proportional to

$$
\Psi_{z}(z=0)=\left(u_{1}-u_{2}\right)(y-\lambda)
$$

which has a positive meridional gradient provided that

$$
u_{1}>u_{2} \text {. }
$$

Note that (4.7b) and (4.11) together imply that $u_{1}$ and $u_{2}$ are both positive.

Now, the dimensionless form of the Charney-Stern theorem (3.4) is

$$
\frac{1}{2}\|\psi(t)\|^{2} \leqslant \max \left\{\frac{C}{c}, \frac{C_{1}}{c_{1}}\right\} \frac{1}{2}\|\psi(0)\|^{2}
$$

(note that the upper-boundary contribution does not enter into the Charney problem, which considers a semi-infinite domain), where

$$
\|\psi\|^{2} \equiv \iint e^{-z / h} c^{-1} \overline{q^{2}} d y d z+\left.\int c_{1}^{-1} \overline{\psi_{z}^{2}} d y\right|_{z=0}
$$

and $c, C, c_{1}$ and $C_{1}$ satisfy

$$
c \leqslant Q_{y} \leqslant C, \quad c_{1} \leqslant \Psi_{z y} \leqslant C_{1} .
$$

As before, (4.12) is equally valid when $c_{i}$ are replaced with $C_{i}$ in (4.13). For the basic flow (4.6), it may be seen from (4.8) and (4.10) that one may take

$$
\begin{gathered}
c=\beta-h^{-1} u_{1}, \quad C=\beta+h^{-1}, \\
c_{1}=C_{1}=u_{1}-u_{2},
\end{gathered}
$$

whence (4.12) may be replaced with

$$
\frac{1}{2}\|\psi(t)\|^{2} \leqslant \frac{\cdot \beta+h^{-1}}{\beta-h^{-1} u_{1}} \frac{1}{2}\|\psi(0)\|^{2} .
$$

\section{a. A bound on the eddy potential enstrophy}

We first seek a bound on the potential enstrophy of the nonzonal part of the flow, namely

$$
\frac{1}{2} \iint e^{-z / h} \overline{P^{\prime 2}}(t) d y d z,
$$

where the prime denotes the deviation from the zonal average. Note that $P^{\prime}=q^{\prime}$ because $Q^{\prime}=0$. Using the fact that $\overline{q^{\prime 2}} \leqslant \overline{q^{2}}$ (by orthogonality of zonal and non- 
zonal flow components), together with (4.13) and (4.16), one obtains

$$
\begin{aligned}
& \frac{1}{2} \iint e^{-z / h} \overline{P^{\prime 2}}(t) d y d z \leqslant \frac{1}{2} \iint e^{-z / h} \overline{q^{2}}(t) d y d z \\
& \leqslant \frac{1}{2} c\|\psi(t)\|^{2} \leqslant \frac{1}{2} \frac{C}{c} c\|\psi(0)\|^{2} \\
& =\frac{1}{2} \frac{C}{c}\left\{\iint e^{-z / h} \overline{q^{2}(0)} d y d z\right. \\
& \left.\quad+\left.\frac{c}{c_{1}} \int \overline{\psi_{z}^{2}(0)} d y\right|_{z=0}\right\} \quad(4.17 \mathrm{a}) \\
& =\frac{1}{2}\left(\beta+h^{-1}\right)\left\{\left(\beta-h^{-1} u_{1}\right)^{-1} \iint e^{-z / h} \overline{q^{2}(0)} d y d z\right. \\
& \left.\quad+\left.\left(u_{1}-u_{2}\right)^{-1} \int \overline{\psi_{z}^{2}(0)} d y\right|_{z=0}\right\}
\end{aligned}
$$

At this point we assume the nonzonal disturbance to be initially of infinitesimal amplitude, so that $\left|q^{\prime}(0)\right|$ $\ll|q(0)|$ and $\left|\psi_{z}^{\prime}(0)\right| \ll\left|\psi_{z}(0)\right|$; hence the initial disturbance to the basic flow (4.6) is dominated by its zonal-mean component. (This assumption will be relaxed later in the section.) Then using (4.4) and (4.8) to find $\bar{q}(0)$, and (4.3) and (4.10) to find $\bar{\psi}_{z}(0)$, we have

$$
\begin{aligned}
& q(0) \approx \bar{q}(0)=\bar{P}(t=0)-Q \\
& =\left\{\begin{array}{l}
h^{-1}\left(u_{1}+1\right)(y-\lambda), \text { for } 0 \leqslant z \leqslant b \\
0, \text { for } z \geqslant b,
\end{array}\right. \\
& \begin{aligned}
\psi_{z}(0) & \approx \bar{\psi}_{z}(0)=\bar{\Phi}_{z}(t=0)-\Psi_{z} \\
= & \left(u_{2}-u_{1}-1\right)(y-\lambda) \text { at } z=0 .
\end{aligned}
\end{aligned}
$$

Combining (4.17b), (4.18) and (4.19) then gives the bound

$$
\begin{aligned}
\frac{1}{2} \iint e^{-z / h} \overline{P^{\prime 2}}(t) d y d z \leqslant \frac{1}{2}\left(\beta+h^{-1}\right) \\
\quad \times\left\{\frac{\left(u_{1}+1\right)^{2}}{h\left(\beta h-u_{1}\right)} \int_{0}^{b} \int_{0}^{1} e^{-z / h}(y-\lambda)^{2} d y d z\right. \\
\left.\quad+\frac{\left(u_{2}-u_{1}-1\right)^{2}}{\left(u_{1}-u_{2}\right)} \int_{0}^{1}(y-\lambda)^{2} d y\right\}
\end{aligned}
$$

It is now time to choose the constant of integration $\lambda$, which arose in the definition of the streamfunction. In particular, $\lambda$ may be chosen so as to minimize the righthand side of (4.20), as this will yield the best bound. It is easily seen that this choice is $\lambda=1 / 2$, which gives $\int_{0}^{1}(y-\lambda)^{2} d y=1 / 12$. Then performing the integration in both $y$ and $z,(4.20)$ takes the form

$$
\begin{aligned}
& \frac{1}{2} \iint e^{-z / h} \overline{P^{\prime 2}}(t) d y d z \leqslant \frac{1}{24}\left(\beta+h^{-1}\right) \\
& \times\left\{\frac{\left(u_{1}+1\right)^{2}}{\left(\beta h-u_{1}\right)}\left(1-e^{-b / h}\right)+\frac{\left(u_{2}-u_{1}-1\right)^{2}}{\left(u_{1}-u_{2}\right)}\right\} \\
& \quad=F_{1}\left(u_{1}, u_{2}, b\right)
\end{aligned}
$$

Equation (4.21) represents a rigorous upper bound on the eddy potential enstrophy (in the limit of an initially infinitesimal nonzonal disturbance), for any choice of the basic-flow parameters $u_{1}, u_{2}$ and $b$ satisfying the stability constraints (4.9) and (4.11) and the matching conditions (4.7). However, the object is now to minimize $F_{1}\left(u_{1}, u_{2}, b\right)$ over all such choices. Since the parameter $u_{0}$ does not enter in (4.21), the constraint (4.7a) may simply be regarded as determining $u_{0}$ and otherwise ignored; this leaves $(4.7 \mathrm{~b}),(4.9),(4.11)$ as constraints on the three free parameters $u_{1}, u_{2}$ and $b$.

The parametric domain is as follows. From (4.9) and (4.1.1), $u_{2}$ must lie in the range

$$
0<u_{2}<\beta h \text {; }
$$

then for fixed $u_{2}, u_{1}$ must lie in the range

$$
u_{2}<u_{1}<\beta h \text {, }
$$

whence (4.7b) implies that $b$ must satisfy

$$
\frac{u_{2}+1}{u_{2}}<e^{b / h}<\frac{\beta h+1}{u_{2}} .
$$

One possible strategy is to eliminate $u_{1}$ using (4.7b), and then seek a local minimum by setting both $\partial F_{1} /$ $\partial u_{2}=0$ and $\partial F_{1} / \partial b=0$. However, trying to solve both equations simultaneously leads to a contradiction. Consequently, one is led to suspect that the minimum of $F_{1}$ occurs at the edge of the parametric domain. Because $F_{1}$ diverges as $u_{2} \rightarrow \beta h$ (since then $u_{1} \rightarrow \beta h$ as well), it is natural to consider the asymptotic limit

$$
u_{2} \rightarrow 0, \quad b / h \rightarrow \infty
$$

(the second condition following from the first by virtue of $(4.7 \mathrm{~b})$ ), in which case $F_{1}$ takes the form

$$
F_{1} \approx \frac{1}{24}\left(\beta+h^{-1}\right)\left\{\frac{\left(u_{1}+1\right)^{2}}{\left(\beta h-u_{1}\right)}+\frac{\left(u_{1}+1\right)^{2}}{u_{1}}\right\},
$$

which is a function of $u_{1}$ alone. It is then a simple matter to minimize (4.25), which gives

$$
\left.F_{1}\right|_{\min }=\frac{1}{6} \frac{(\beta h+1)^{2}}{\beta h^{2}}
$$

for the choice $u_{1}=\beta h /(\beta h+2)$. Note that this choice of $u_{1}$ does indeed satisfy both (4.9) and (4.11).

The minimum implied by (4.26) may be approached arbitrarily closely for small but nonzero $u_{2}$ and large but finite $b$. The result was checked numerically by computing $F_{1}\left(u_{1}, u_{2}, b\right)$ for fixed $u_{2}$ over the range of 
$b$ given by (4.23) (after eliminating $u_{1}$ ), and varying $u_{2}$ over the range (4.22). It was found that, indeed, the minimum of $F_{1}$ over $b$ (for given $u_{2}$ ) decreased as $u_{2}$ decreased, and approached the limit (4.26) as $u_{2} \rightarrow 0$.

However, there is one rather curious aspect of the above derivation, which offers scope for improvement on the bound (4.26). The expression (4.25) for $F_{1}\left(u_{1}\right)$ still retains the factor $\beta+h^{-1}$, representing the maximum basic-state potential-vorticity gradient, despite the fact that in the limit $b / h \rightarrow \infty$ under which (4.25) was derived, this value of $Q_{y}$ is found only at heights $z \geqslant b \gg h$, where it would seem to be irrelevant to the dynamics of the instability. Indeed, the factors $C / c$ and $C / c_{1}$ which are present in (4.25) [ see (4.17)] take values of

$$
\begin{aligned}
& \frac{C}{c}=\frac{\beta+h^{-1}}{\beta-h^{-1} u_{1}}=\frac{\beta h+2}{\beta h}, \\
& \frac{C}{c_{1}}=\frac{\beta+h^{-1}}{u_{1}}=\frac{(\beta h+1)(\beta h+2)}{\beta h^{2}}
\end{aligned}
$$

for the choice $u_{1}=\beta h /(\beta h+2)$, both of which are $O(1 / \beta h) \gg 1$ in the limit $\beta h \rightarrow 0$. Certainly in this latter regime, then, the fact that we are forced to retain $C=\beta+h^{-1}$, despite its apparent dynamical irrelevance, is evidently very damaging.

All this suggests that one may significantly improve upon the bound (4.26) by eliminating all reference to $\beta+h^{-1}$. This can be done by taking a rather drastic step, namely by setting $b=\infty$ and $u_{2}=0$ exactly, and abandoning the matching conditions (4.7) altogether. That is, for the basic flow one chooses (4.6) with $b$ $=\infty$ and $u_{2}=0$, namely

$$
U(z)=-u_{1} z
$$

through the depth of the atmosphere. (The extraneous parameter $u_{0}$ can now be safely set to zero.) While this may seem like an odd choice, insofar as it diverges from the initial zonal-mean flow (4.3) as $z \rightarrow \infty$, the point is that the initial disturbance potential vorticity $q(0)$ (which is still given by (4.18), but with $b=\infty$ ) is independent of $z$, and is thus controlled by the exponentially-decreasing density factor. The advantage of taking (4.27) as the basic flow is that now $Q_{y}$ is a constant throughout the domain, and consequently

$$
C=c=\beta-h^{-1} u_{1}
$$

rather than (4.15a). In particular, $C / c=1$.

At this stage, it is easy to allow for the case of a finiteamplitude initial nonzonal disturbance. Denoting the initial eddy potential enstrophy and eddy APE respectively by $Z_{0}$ and $Y_{0}$, viz.

$$
\begin{gathered}
Z_{0} \equiv \frac{1}{2} \iint e^{-z / h} \overline{P^{\prime 2}}(0) d y d z, \\
\left.Y_{0} \equiv \frac{1}{2} \int \overline{\Phi_{z}^{\prime 2}}(0) d y\right|_{z=0},
\end{gathered}
$$

using (4.18) and (4.19) to evaluate the zonal-mean disturbance contribution at $t=0$, and taking (4.15b) and $(4.28)$, the bound (4.17a) on the eddy potential enstrophy becomes

$$
\begin{array}{r}
\frac{1}{2} \iint e^{-z / h} \overline{P^{\prime 2}}(t) d y d z \leqslant \frac{\beta}{24} \frac{\left(u_{1}+1\right)^{2}}{u_{1}}+Z_{0} \\
+\frac{\beta-h^{-1} u_{1}}{u_{1}} Y_{0} \equiv \tilde{F}_{1}\left(u_{1} ; Z_{0}, Y_{0}\right)
\end{array}
$$

This bound is valid for arbitrary $Y_{0}$ and $Z_{0}$, and is to be considered over the range (4.9), viz. $0<u_{1}<\beta h$. It takes a local minimum at $u_{1}=\eta \equiv\left(24 Y_{0}+1\right)^{1 / 2}$, but this only occurs within the permissible range of $u_{1}$ for $\beta h>\eta$. When $\beta h \leqslant \eta$, the minimum of (4.30) compatible with (4.9) is achieved in the limit $u_{1} \rightarrow$ $\beta h$. In both cases, when $Z_{0}, Y_{0} \rightarrow 0$ the resulting bound is smaller than (4.26): for $\beta h \gg 1$ the two bounds are in fact asymptotically equal, but for $\beta h \ll 1$ the bound derived from ( 4.30 ) is equal to $\beta h / 4$ times ( 4.26 ), and is thus much smaller.

One may therefore write down the general bound on the eddy potential enstrophy, obtained from the nonlinear stability theorem (4.16), as

$$
\begin{aligned}
& \frac{1}{2} \iint e^{-z / h} \overline{P^{\prime 2}}(t) d y d z \\
& \quad \leqslant \begin{cases}\frac{\beta}{12}(\eta+1)+Z_{0}-h^{-1} Y_{0}, & \text { for } \beta h \geqslant \eta \\
\frac{1}{24} \frac{(\beta h+1)^{2}}{h}+Z_{0}, & \text { for } \beta h \leqslant \eta\end{cases}
\end{aligned}
$$

with $\eta \equiv\left(24 Y_{0}+1\right)^{1 / 2}$, and $Y_{0}, Z_{0}$ defined by (4.29). In the important special case of an initial nonzonal disturbance of infinitesimal amplitude (i.e. the limit $\left.Y_{0} \rightarrow 0, Z_{0} \rightarrow 0\right), \eta \rightarrow 1$ and the bound simplifies to $\frac{1}{2} \iint e^{-z / h} \overline{P^{\prime 2}}(t) d y d z$

$$
\leqslant\left\{\begin{array}{l}
\frac{1}{6} \beta, \text { for } \beta h \geqslant 1 \\
\frac{1}{24} \frac{(\beta h+1)^{2}}{h}, \text { for } \beta h \leqslant 1 .
\end{array}\right.
$$

Now, to this point we have not considered the fact that there is another constraint on the eddy potential enstrophy, namely that it cannot exceed the total amount of potential enstrophy in the system. The bound given by (4.31) is clearly useless if it exceeds this latter quantity, so it is important to determine just how much potential enstrophy is, in principle, available to the eddies, quite apart from stability considerations.

Because the potential vorticity $P$ is materially conserved for each $z$, it follows that the potential enstrophy at each level, $\frac{1}{2} \int \overline{P^{2}} d y$, is invariant 
in time. Since $\int \bar{P} d y$ is likewise invariant, it follows that $\int \overline{(P-\mu)^{2}} d y$ is invariant for any constant $\mu$. It is then possible to bound the eddy potential enstrophy according to

$$
\begin{aligned}
\frac{1}{2} \iint e^{-z / h} \overline{P^{\prime 2}} d y d z & =\frac{1}{2} \iint e^{-z / h} \overline{(P-\mu)^{2}} d y d z \\
& -\frac{1}{2} \iint e^{-z / h}(\bar{P}-\mu)^{2} d y d z \\
\leqslant & \frac{1}{2} \iint e^{-z / h} \overline{(P-\mu)^{2}} d y d z
\end{aligned}
$$

At $t=0$, the invariant

$$
Z \equiv \frac{1}{2} \iint e^{-z / h} \overline{(P-\mu)^{2}} d y d z
$$

consists of the initial eddy potential enstrophy $Z_{0}$ plus a zonal-mean part which may be determined directly from (4.4). It is advantageous at this stage to choose $\mu$ so as to minimize $Z$, which for the Charney problem is achieved by taking $\mu \equiv \bar{P}(y=1 / 2)=f+\beta / 2$ (this presumes the choice $\lambda=1 / 2$, which makes the zonalmean relative potential vorticity vanish at the channel midpoint $y=1 / 2$ ). With this value of $\mu$, and using (4.4), $Z$ is given by

$$
\begin{aligned}
\bar{Z} & =Z_{0}+\frac{1}{2} \iint e^{-z / h}\left(\beta+h^{-1}\right)^{2}\left(y-\frac{1}{2}\right)^{2} d y d z \\
& =\frac{1}{24} \frac{(\beta h+1)^{2}}{h}+Z_{0},
\end{aligned}
$$

which is identical to (4.31) for $\beta h \leqslant \eta$. When $\beta h>\eta$, $Z$ exceeds (4.31); for the case $Y_{0}, Z_{0} \rightarrow 0$ it does so by a factor $\beta h / 4$ in the limit $\beta h \rightarrow \infty$. Therefore, when $\beta h>\eta$, the rigorous upper bound (4.31) derived by using the Charney-Stern theorem limits the eddy potential enstrophy below the maximum amount in the system. To put it another way, the Charney-Stern theorem provides a useful constraint on the flow dynamics, in the sense that it prevents the eddies from growing to the size permitted by the global constraint (4.33), only for $\beta h>\eta$. The larger the initial eddy amplitude, the larger the value of $\beta h$ that is required for this.

In terms of dimensional variables, the bound (4.31) takes the form

$$
\begin{aligned}
& \frac{1}{2} \iint \rho \overline{P^{\prime 2}}(t) d y d z \\
& \quad \leqslant\left\{\begin{array}{rrr}
\frac{1}{12} \rho_{0} \beta_{0}{ }^{2} H L^{3} \gamma(\eta+1)+Z_{0}^{*} \\
-f_{0}^{2} g^{2} H^{-1} N^{-4} T_{0}{ }^{-2} Y_{0}^{*}, & \text { for } & \gamma \leqslant \eta^{-1} \\
\frac{1}{24} \rho_{0} \beta_{0}{ }^{2} H L^{3}(1+\gamma)^{2}+Z_{0}^{*}, & \text { for } \gamma \geqslant \eta^{-1},
\end{array}\right.
\end{aligned}
$$

where

$$
\begin{aligned}
& Z_{0}^{*}=\rho_{0} L D(\xi D / L)^{2} Z_{0}=\rho_{0}\left(f_{0} / N\right)^{3} L^{2} \xi^{2} Z_{0}, \\
& Y_{0}^{*}=\rho_{0} L\left(T_{0} f_{0} / g\right)^{2}(\xi L)^{2} Y_{0}
\end{aligned}
$$

are the dimensional initial eddy potential enstrophy and eddy APE, and

$$
\gamma \equiv \frac{1}{\beta h}=\frac{f_{0}^{2} \xi}{\beta_{0} N^{2} H} .
$$

Green (1960) and Held (1978) have shown that $\gamma$ (which equals $h / H$ in Held's notation) is a crucial dimensionless parameter determining the nature of the linearly most unstable wave in the Charney problem. In the limit $\gamma \gg 1$, the relevant vertical scale for the instability is the scale height $H$, and the horizontal scale $N H / f_{0}$, as in the Eady (1949) model of baroclinic instability on an $f$-plane $\left(\beta_{0}=0\right)$. But in the limit $\gamma$ $\ll 1$, which may be considered a Boussinesq limit, the relevant vertical scale is instead

$$
\gamma H=\frac{f_{0}^{2} \xi}{\beta_{0} N^{2}},
$$

which has a linear dependence on the vertical shear. To estimate $\gamma$ for the midlatitude troposphere, one may consider values of $f_{0} \approx 10^{-4} \mathrm{~s}^{-1}, \beta_{0} \approx 1.5 \times 10^{-11}$ $\mathrm{m}^{-1} \mathrm{~s}^{-1}, N^{2} \approx 2 \times 10^{-4} \mathrm{~s}^{-2}, H \approx 8 \mathrm{~km}$, and $\xi \approx 50$ $\mathrm{m} \mathrm{s}^{-1} / 10 \mathrm{~km}=5 \times 10^{-3} \mathrm{~s}^{-1}$, which gives $\gamma \approx 2$ or $\beta h \approx 0.5$.

\section{b. $A$ bound on the eddy $A P E$ at $z=0$}

We now seek a bound on the quantity

$$
\left.\frac{1}{2} \int \overline{\Phi_{z}^{\prime 2}}(t) d y\right|_{z=0} .
$$

In this case it turns out that the tightest bound comes from using the norm given by (4.13) with $c_{i}$ replaced by $C_{i}$. Noting that $\Phi^{\prime}=\psi^{\prime}$ because $\Psi^{\prime}=0$, and using (4.16) with this alternative norm,

$$
\begin{array}{r}
\left.\frac{1}{2} \int \overline{\Phi_{z}^{\prime 2}}(t) d y\right|_{z=0} \leqslant\left.\frac{1}{2} \int \overline{\psi_{z}^{2}}(t) d y\right|_{z=0} \leqslant \frac{1}{2} C_{1}\|\psi(t)\|^{2} \\
\leqslant \frac{1}{2} \frac{C}{c} C_{1}\|\psi(0)\|^{2}=\frac{1}{2} \frac{C}{c}\left\{\frac{C_{1}}{C} \iint e^{-z / h} \overline{q^{2}(0)} d y d z\right. \\
\left.+\left.\int \overline{\psi_{z}^{2}(0)} d y\right|_{z=0}\right\}
\end{array}
$$

As with the potential-enstrophy bound, it turns out that one does best by considering the basic flow (4.27), with (4.15b) and (4.28). Then using the definitions (4.29), together with the right-hand sides of (4.18) and (4.19) to evaluate the zonal-mean disturbance contribution at $t=0$, the bound (4.38) takes the form 


$$
\begin{aligned}
\left.\frac{1}{2} \int \overline{\Phi_{z}^{\prime 2}}(t) d y\right|_{z=0} \leqslant \frac{\beta h}{24} \frac{\left(u_{1}+1\right)^{2}}{\beta h-u_{1}}+Y_{0} \\
+\frac{u_{1} h}{\beta h-u_{1}} Z_{0} \equiv \tilde{F}_{2}\left(u_{1} ; Z_{0}, Y_{0}\right)
\end{aligned}
$$

Now, $\tilde{F}_{2}$ takes local minima at $u_{1}=\beta h \pm\left((\beta h+1)^{2}\right.$ $\left.+24 h Z_{0}\right)^{1 / 2}$, both of which are outside the permissible range of $u_{1}$, viz. $0<u_{1}<\beta h$. The minimum of (4.39) over this range is achieved in the limit $u_{1} \rightarrow 0$, and is simply

$$
\left.\tilde{F}_{2}\right|_{\min }=\frac{1}{24}+Y_{0} .
$$

As with the eddy potential enstrophy, it is important to compare the bound (4.40) on the eddy APE at the surface with any global constraint in the full problem. In fact, the material conservation of $\Phi_{z}$ at $z=0$ expressed by (2.4) implies that $\int \Phi_{z}{ }^{2} d y$ at $z=0$ is invariant, and therefore the eddy APE is bounded according to

$$
\left.\frac{1}{2} \int \overline{\Phi_{z}^{\prime 2}}(t) d y\right|_{z=0} \leqslant\left.\frac{1}{2} \int \overline{\Phi_{z}^{2}}(t) d y\right|_{z=0} .
$$

This last integral is obtainable at $t=0$ for the Charney problem using (4.3) for the zonal-mean contribution and $Y_{0}$ for the eddy contribution, yielding

$$
\left.\frac{1}{2} \int \overline{\Phi_{z}^{\prime 2}}(t) d y\right|_{z=0} \leqslant \frac{1}{24}+Y_{0}
$$

which is identical to (4.40). In terms of dimensional variables, the bound becomes

$$
\left.\frac{1}{2} \int \rho \overline{\theta^{\prime 2}} d y\right|_{z=0} \leqslant \frac{1}{24} \rho_{0} T_{0}^{2} f_{0}^{2} \xi^{2} L^{3} g^{-2}+Y_{0}^{*}
$$

\section{Comparison with baroclinic-adjustment arguments}

When trying to estimate the stage at which baroclinic instability will saturate, the concept of baroclinic adjustment (Stone 1978; Lindzen and Farrell 1980) is intuitively appealing. The hypothesis involved here is that the disturbance will grow only until such time as the zonal-mean state is neutralized to linear instability. There are, of course, many difficulties with this sort of argument, which is in any case essentially ad hoc. In particular, it is based on linear theory, whereas by the time the "adjusted" state is reached the disturbance is of finite amplitude; indeed, in weakly nonlinear theory for the two-layer model, Pedlosky (1970) found that the disturbance continued to grow past the point where the zonal-mean flow was neutralized, rendering the latter subcritical when the disturbance finally saturated. Moreover, in forced-dissipative problems the equilibrated zonal flow is generally substantially supercritical in realistic parameter regimes (Salmon 1980; James 1987; Vallis 1988).
Nevertheless, in unforced problems of the type under consideration here, baroclinic adjustment may be regarded as providing a plausible estimate of maximum disturbance amplitudes. Therefore, it is of interest to determine the minimum amount of potential enstrophy and available potential energy that must be released by the zonal flow in order to render itself neutral.

Consider first the eddy potential enstrophy, which may be computed under the neutralization hypothesis from $(4.33 \mathrm{a})$. A particularly simple neutral state is in fact found by taking the zonal-mean flow to equal the basic flow (4.6), (4.8), but with the inequalities (4.9) and (4.11) replaced with equalities so that $Q_{y}=0$ for $z \leqslant b$ and $\Psi_{z y}=0$ at $z=0$. (This corresponds to the neutral state considered by Lindzen and Farrell 1980.) These two conditions, together with $(4.7 \mathrm{~b})$, are then enough to determine the three parameters $u_{1}, u_{2}$, and $b$ : in particular,

$$
u_{1}=u_{2}=\beta h, \quad e^{b / h}=(\beta h+1) / \beta h .
$$

For this neutralized zonal-mean flow, with $\bar{P}(y, z)$ given by (4.8) and (5.1), and taking $\lambda=1 / 2$ and $\mu=f$ $+\beta / 2$ as before, the third integral in (4.33a) can be evaluated according to

$$
\begin{aligned}
\frac{1}{2} \iint e^{-z / h}(\tilde{P}-\mu)^{2} d y d z \\
=\frac{1}{24}\left\{\int_{0}^{b} e^{-z / h}\left(\beta-h^{-1} u_{1}\right)^{2} d z\right. \\
\left.\quad+\int_{b}^{\infty} e^{-z / h}\left(\beta+h^{-1}\right)^{2} d z\right\} \\
=\frac{1}{24}\left(\beta+h^{-1}\right)^{2} h e^{-b / h}=\frac{1}{24} \beta(\beta h+1)
\end{aligned}
$$

then (4.33a), (4.34), (4.35) and (5.2) imply that

$$
\frac{1}{2} \iint e^{-z / h} \overline{P^{\prime 2}} d y d z=\frac{1}{24} \frac{\beta h+1}{h}+Z_{0} .
$$

Equation (5.3) represents one estimate of the saturated value of the eddy potential enstrophy according to baroclinic adjustment arguments, and is to be compared with the rigorous upper bound given by (4.31).

In the limit $\beta h \gg 1$ [or $\gamma \ll 1$, for $\gamma$ defined by (4.37)], corresponding to "shallow" disturbances, the ratio of the two quantities is given by

$$
\frac{\text { Eq. }(4.31)}{\text { Eq. }(5.3)} \approx \frac{2 \beta(\eta+1)+24 Z_{0}}{\beta+24 Z_{0}} .
$$

Both are much smaller than the total available in the system, (4.35). In the limit $Y_{0}, Z_{0} \rightarrow 0,(5.4) \rightarrow 4$ in which case the upper bound is simply four times the adjustment estimate. There is an interesting parallel here with the case of the barotropic "point-jet" instability (Shepherd 1988b, §7.3): using the same kind of method to determine a rigorous upper bound on the 
eddy enstrophy yields (for the case of an initially infinitesimal wavy disturbance) a value four times the "saturation limit" predicted by Schoeberl and Lindzen (1984) on the basis of presumed adjustment to a neutral profile. In a numerical simulation of single-wave equilibration presented by those authors (op. cit., Fig. 8), the eddy enstrophy actually overshot the saturation limit by a factor of two, coming within a factor of two of the rigorous upper bound.

It may also be pointed out that for this case of $\beta h$ $\gg 1$, the "adjustment depth" $b$ over which the zonalmean flow is presumed to be modified is given according to (5.1) by

$$
b=h \ln \left(1+(\beta h)^{-1}\right) \approx 1 / \beta ;
$$

in dimensional units, this corresponds to

$$
b \approx \frac{D}{\beta}=\frac{f_{0}^{2} \xi}{\beta_{0} N^{2}}=\gamma H
$$

which is indeed the relevant vertical scale in this regime (Held 1978).

In the limit $\beta h \ll 1$ (or $\gamma \gg 1$ ), on the other hand, the adjustment estimate (5.3) approaches the stabilitybased bound (4.31), which in fact equals the global upper bound (4.35). One is therefore led to suspect that the eddies will take up all the potential enstrophy that is available to them.

With regard to the calculation leading to (5.3), it may be objected that, to obtain a neutral zonal-mean profile, there is no need for the zonal-mean potential vorticity gradient $\bar{P}_{y}$ to vanish in some layer, as assumed by (5.1); allowing it to be reduced from that of the Charney profile (4.4), namely $\beta+h^{-1}$, to some smaller positive value would clearly suffice. Consequently, it is of interest to consider a more general class of neutral profiles than that implied by (5.1).

This class is again found by taking the zonal-mean flow to equal the basic flow (4.6), (4.8). Because it is still natural to assume that the surface-temperature gradient is eliminated in a neutral profile, $(4.11)$ is again taken to be an equality; but (4.9) is this time left as an inequality. Instead of (5.1), then, one has

$$
u_{1}=u_{2}, \quad e^{b / h}=\frac{u_{1}+1}{u_{1}}
$$

with $u_{1}$ left free, though with $0<u_{1} \leqslant \beta h$ to satisfy (4.9). The first equation of (5.2) may be used here, which then gives for such profiles

$$
\begin{aligned}
& \frac{1}{2} \iint e^{-z / h}(\bar{P}-\mu)^{2} d y d z \\
& =\frac{1}{24} h^{-1}\left\{\left(\beta h-u_{1}\right)^{2}\left(1-e^{-b / h}\right)\right. \\
& \left.\quad+(\beta h+1)^{2} e^{-b / h}\right\}=\frac{1}{24} h^{-1}\left(\beta^{2} h^{2}+u_{1}\right),
\end{aligned}
$$

after using (5.6) to eliminate $e^{b / h}$. To allow the greatest possible eddy potential enstrophy, one seeks to minimize the right-hand side of (5.7) over all suitable values of $u_{1}$; this is evidently achieved in the limit $u_{1} \rightarrow 0$. Then using (5.7) with $u_{1} \rightarrow 0$, together with (4.33a), (4.34) and (4.35), one has

$$
\frac{1}{2} \iint e^{-z / h} \overline{P^{\prime 2}} d y d z=\frac{1}{24} \frac{2 \beta h+1}{h}+Z_{0},
$$

which is larger than (5.3). In the limit $\beta h \gg 1$ and $Y_{0}$, $Z_{0} \rightarrow 0,(5.8)$ is twice (5.3); for $\beta h \ll 1$, they are equivalent. It is perhaps worth emphasizing again that both (5.3) and (5.8) are no more than ad hoc estimates of the maximum eddy potential enstrophy under various neutralization hypotheses; moreover, (5.8) is evidently somewhat unphysical insofar as the "adjustment depth" $b$ is presumed to be infinite.

Figure 2 compares the rigorous upper bound (4.31), the maximum permitted eddy potential enstrophy (4.35), and the two adjustment estimates (5.3) and (5.8), over a wide range of $\beta h$ for the case $Y_{0}, Z_{0} \rightarrow$ 0 and $\eta \rightarrow 1$. For $\beta h \ll 1$, all the curves are virtually coincident; for $\beta h \gg 1$, however, there is a fanning out, with $(4.31):(5.8):(5.3)$ in the ratio $4: 2: 1$, and all three much smaller than (4.35). This latter regime is clearly

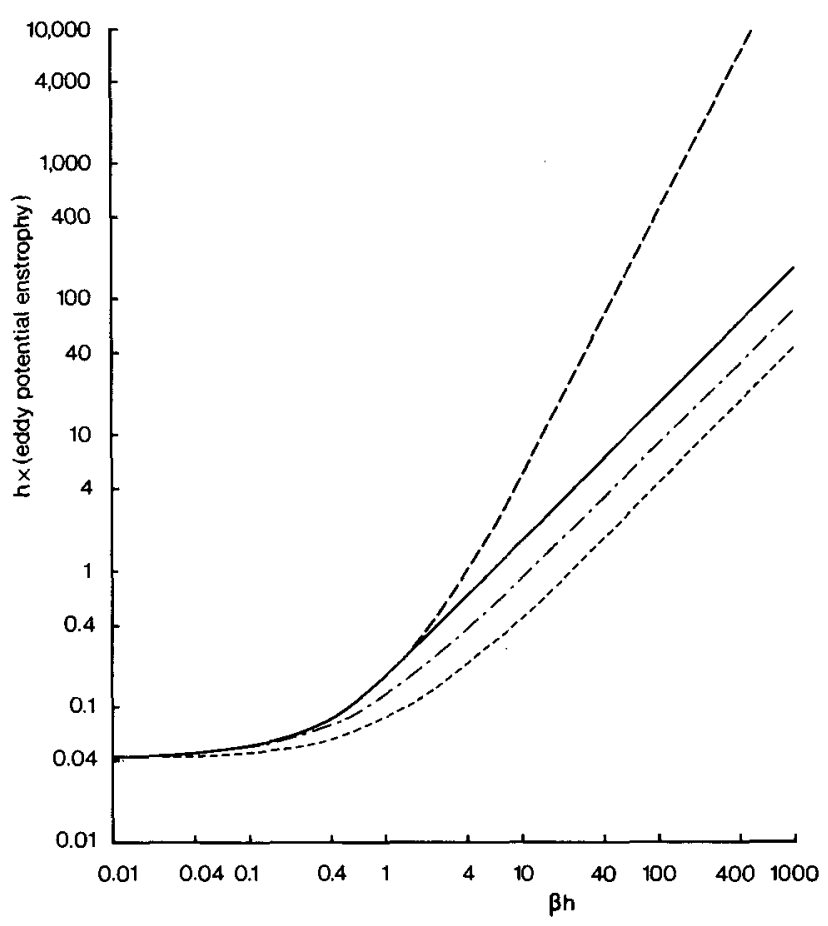

FIG. 2. Comparison of the rigorous upper bound on the eddy potential enstrophy (4.31) obtained from stability considerations (solid curve), the (minimum value of the) total potential enstrophy in the system (4.35) (long-dashed curve), and the two baroclinic-adjustment estimates (5.3) (short-dashed curve) and (5.8) (dash-dot curve), in terms of $\beta h=\gamma^{-1}$ [see (4.37)]; all for the limit $Y_{0}, Z_{0} \rightarrow 0$ corresponding to nonzonal disturbances of infinitesimal initial amplitude. 
the one of most interest in the present context, and a comparison of these results with weakly-nonlinear theory for $\beta h \gg 1$ is therefore attempted in the next section.

The situation with regard to the eddy APE at $z$ $=0$ can be dealt with quickly. Because a neutralized state must have $\bar{\Phi}_{z y}=0$ at $z=0$, and thus no zonal APE (assuming that $\bar{\Phi}_{z}$ vanishes for some $y \in\left[y_{1}\right.$, $y_{2}$ ], as it does for $\bar{\Phi}_{z}$ given by (4.10)), it follows that baroclinic adjustment must always predict eddy APE at $z=0$ equal to the total amount available, namely (4.42) with the inequality replaced by an equality. Since the stability-based bound (4.40) gives the same result, one is led to suspect that the saturated state will indeed be one with all the APE at $z=0$ in the eddies. The numerical experiments of Simmons and Hoskins (1978) indeed suggest that, at least in the absence of surface damping, saturation does involve the expulsion of the surface temperature gradient within the jet region. (When $\bar{\Phi}_{z}$ is not taken to vanish at some $y \in\left[y_{1}, y_{2}\right]$, then it is only the minimum of $\left.\frac{1}{2} \int \overline{\left(\Phi_{z}-\mu\right)^{2}} d y\right|_{z=0}$ - which is an invariant-over all constants $\mu$ which is available to the eddies. For the discussion above, however, the minimum of this quantity obtains for $\mu=0$.)

\section{Comparison with single-wave equilibration from weakly nonlinear theory for $\beta h \gg 1$}

The weakly nonlinear theory of Pedlosky (1979b) provides an explicit prediction of the time evolution of a single-wave disturbance in the Charney model of baroclinic instability. For given external parametersin particular for given $\beta$, which may be considered a dimensionless measure of the inverse vertical shear $\xi^{-1}$ - one may therefore seek to determine the maximum eddy potential enstrophy predicted by the weakly nonlinear theory for a given wave, and compare this with the various quantities shown in Fig. 2. (It may be noted that Pedlosky's $1979 \mathrm{~b}$ analysis employs the same nondimensionalization as that used in this paper.)

Unlike the case of the two-layer model, there is of course no stability threshold in the Charney model. However, Pedlosky (1979b) focused on the so-called Charney modes, for which a given wavenumber $\kappa$ $\equiv\left(k^{2}+\pi^{2} l^{2}\right)^{1 / 2}$ (where $k$ and $\pi l$ are respectively the zonal and meridional wavenumbers ) has a critical shear given by

$$
\beta=\beta_{c}(\kappa) \equiv\left(h^{-2}+4 \kappa^{2}\right)^{1 / 2}-h^{-1} .
$$

(Pedlosky used $\beta_{0}$ rather than $\beta_{c}$, but we retain the latter to avoid possible confusion with the dimensional beta parameter.) The theory assumes that the supercriticality $\Delta(\kappa)$, given by

$$
\Delta(\kappa) \equiv \beta_{c}(\kappa)-\beta,
$$

is small, and it is the square root of this parameter $\Delta(\kappa)$ which is taken as the expansion parameter in the perturbation series. The situation is shown schematically in Fig. 3, the key point to note being the absence of any minimum critical shear (or maximum $\beta_{c}$ ). For $\beta$ $>\beta_{c}(\kappa)$ the flow is still susceptible to normal-mode instability at scale $\kappa$ (except for a countable set of values corresponding to neutral modes), but these instabilities are rather weaker than those of the Charney modes and are not considered in Pedlosky's analysis.

The relevant case to consider for present purposes is that of nearly inviscid flow (Pedlosky $1979 \mathrm{~b}$, $\$ 4$, with his $\gamma \rightarrow 0$ ) and $\beta h \gg 1$; we also simplify the discussion by restricting attention to the case of infinitesimal initial disturbance amplitude. To leading order in $|\Delta|^{1 / 2}$, the potential vorticity of the nonzonal part of the flow is given according to the weakly nonlinear theory by

$$
\begin{aligned}
q^{\prime}=-\frac{\left(\beta_{c}+h^{-1}\right)}{z} \psi^{\prime}=- & |\Delta|^{1 / 2}\left(\beta_{c}+h^{-1}\right) e^{-\beta_{c} z / 2} 2 \\
& \times \operatorname{Re}\left[A e^{i \theta} \sin (\pi l y)\right]
\end{aligned}
$$

[Pedlosky 1979b, Eqs. (3.14) and (3.28)], where $\theta$ is the phase. The maximum of the amplitude $|A|^{2}$ is predicted to be

$$
|A|_{\max }^{2}=\frac{\pi^{2} a_{0}}{q_{0}^{3} l^{4} D\left(a_{0}, l\right)} R_{\max }^{2},
$$

where $R_{\max }^{2}=2$ for $R(0) \ll 1, a_{0}=\kappa / \pi, q_{0} \equiv \beta_{c}$ $+h^{-1}$, and $D\left(a_{0}, l\right)$ is a complicated function which is given by Pedlosky's equation (4.17b). For fixed $a_{0}$ (and $k$ ), $l^{4} D\left(a_{0}, l\right)$ is minimized for the gravest meridional mode, $l=1$, so this choice of $l$ is made here so as to make $|A|^{2}$ as large as possible. Taking $\beta_{c}$ $\geqslant \beta \gg h^{-1}$ in (6.3) and (6.4), so that $\beta_{c}(\kappa) \approx 2 \kappa$ $=2 \pi a_{0}$, the maximum eddy potential enstrophy for a given $a_{0}$ therefore takes the form

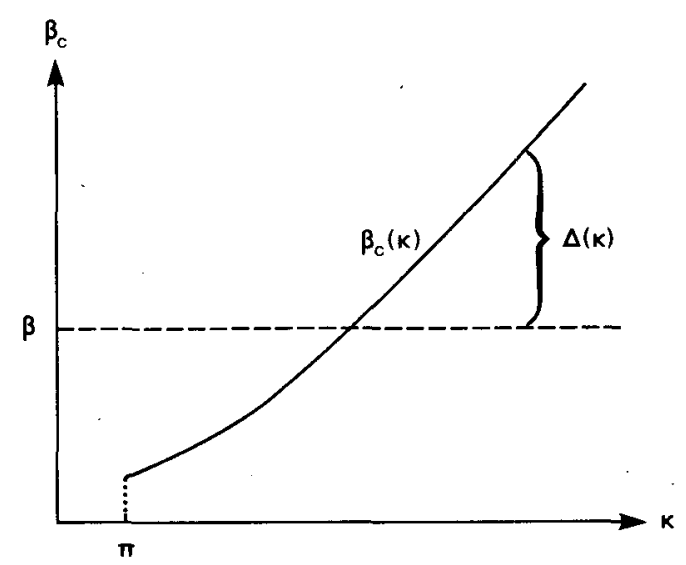

FIG. 3. Schematic of the marginal stability curve $\beta_{c}(\kappa)$ for the Charney modes, which is given by (6.1); for large $\alpha$ (small horizontal scale) the supercriticality $\Delta(\kappa)$ becomes increasingly large. 


$$
\begin{aligned}
\frac{1}{2} \iint e^{-z / h} \overline{q^{\prime 2}} d y d z & =\frac{1}{2}|\Delta| \beta_{c}{ }^{2}|A|_{\max }^{2} \\
& \times \int e^{-\beta_{c^{2}}} d z=\frac{2 \pi a_{0}-\beta}{4 a_{0} D\left(a_{0}\right)} .
\end{aligned}
$$

The correctness of the above expression has been checked by calculating the quantity

$$
\frac{1}{2} \iint e^{-z / h}(\bar{P}-\mu)^{2} d y d z
$$

from weakly nonlinear theory, and then using (4.33a) to determine the maximum eddy potential enstrophy (see appendix B).

The right-hand side of (6.5) is a linear function of $\beta$; its dependence on $a_{0}$ is shown in Fig. 4 for $\beta=10$, and is evidently very nearly linear as well. Note that $a_{0}$ must exceed unity because of the existence of a gravest meridional mode in this channel geometry, and that for $\Delta$ to be positive it is necessary that $a_{0}>\beta / 2 \pi$. The rigorous upper bound (4.32) and the adjustment estimate (5.3) for the case $Y_{0}, Z_{0} \rightarrow 0$ are also indicated in Fig. 4; in this Boussinesq limit of $\beta h \gg 1$ they are given, respectively, by $\beta / 6$ and $\beta / 24$. The leading-order prediction of the weakly nonlinear theory is clearly invalid for $a_{0}>2.3$, when it violates the rigorous upper bound. However, when $a_{0}=2.3$ the supercriticality $\Delta$ $=4.4$, so one would not expect the theory to be particularly reliable there in any case. Indeed, for $\beta=10$, $\Delta>1$ for $a_{0}>1.75$, which suggests that the range of wavenumbers for which weakly nonlinear theory might be relevant is very small indeed. Whereas this range

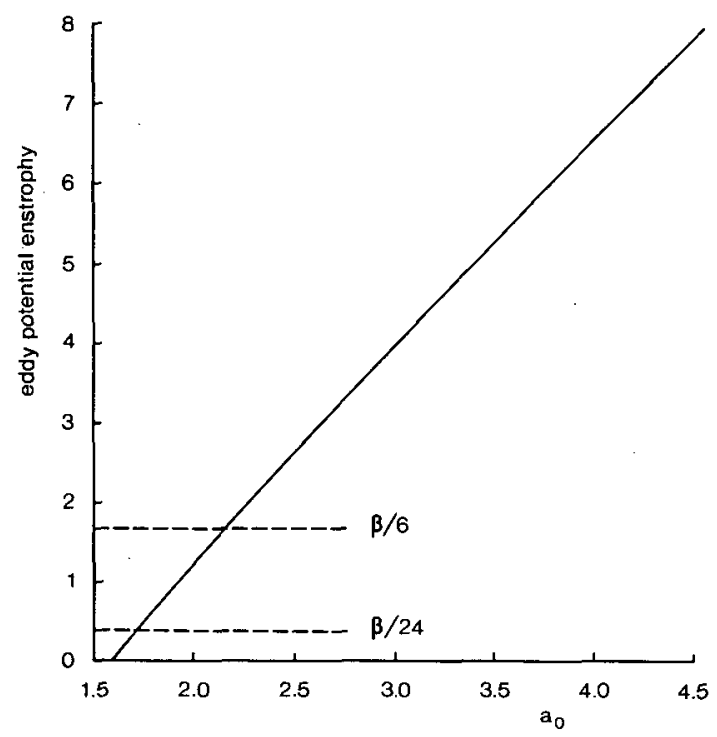

FIG. 4. The maximum eddy potential enstrophy (6.5) predicted by Pedlosky's (1979b) weakly nonlinear theory for $\beta=10$ in the inviscid limit (solid curve), as a function of $a_{0}=\kappa / \pi$, compared with the rigorous upper bound $\beta / 6$ and the baroclinic-adjustment estimate $\beta / 24$. may nevertheless be physically meaningful in a twolayer fluid, because of the existence of a minimum critical shear, its physical significance is less clear for the Charney problem where, for given $\beta$, the flow is supercritical to an arbitrarily large degree in the shortwave (large $\kappa$ ) limit.

These results are summarized in Fig. 5. In the ( $\beta$, $a_{0}$ ) parametric domain, curves have been drawn corresponding to constant values of the supercriticality $\Delta$ and of $r$, the ratio of the maximum eddy potential enstrophy predicted by the weakly-nonlinear theory, (6.5), to the rigorous upper bound (4.32) - the latter being, of course, just $\beta / 6$ in this regime. For $r>1$, the weakly nonlinear theory is in violation of the bound, and must be wrong; Fig. 5 demonstrates that this state of affairs only obtains for $\Delta>1$. When $\Delta<1, r$ is generally at least as small as 0.5 . The region above $\Delta$ $=0$ corresponds to the long-wave "Green modes," and is not covered by the weakly nonlinear analysis. Once again, the small domain of validity for weakly nonlinear theory is highlighted. It is apparent that a meaningful comparison between such theory and the results of sections 4 and 5 is not really possible. This is in contrast to the situation found in Part I for the Phillips model. of baroclinic instability in a two-layer fluid; there the parameter dependences of the exact upper bound and Pedlosky's (1970) weakly nonlinear theory were found to be rather similar.

\section{Nonlinear saturation bounds for more general pro- files}

In this section, we consider the problem of deriving saturation bounds for instabilities to general profiles

$$
\hat{U}(y, z) \equiv-\frac{\partial}{\partial y} \bar{\Phi}(y, z, t=0) .
$$

The first point to make is that for a given initial profile, and for given values of the initial eddy potential enstrophy $Z_{0}^{*}$ and eddy APE $Y_{0}^{*}$, the Charney-Stern theorem (3.4) provides an infinity of bounds-one for each choice of a stable basic state $(\Psi, Q)$. This is because the eddy potential enstrophy (or the eddy APE at $z=0$ ) may be bounded in terms of the left-hand side of (3.4), while the right-hand side of (3.4) is a functional of $\Psi(y, z)$. Specifically, given $(\Phi, P)$ at $t$ $=0$ and $(\Psi, Q)$, one may use

$$
\begin{aligned}
q(t=0) & =\bar{q}(t=0)+q^{\prime}(t=0) \\
& =\bar{P}(t=0)-Q+P^{\prime}(t=0)
\end{aligned}
$$

in the interior, and

$$
\begin{aligned}
\psi_{z}(t=0) & =\bar{\psi}_{z}(t=0)+\psi_{z}^{\prime}(t=0) \\
& =\bar{\Phi}_{z}(t=0)-\Psi_{z}+\Phi_{z}^{\prime}(t=0)
\end{aligned}
$$

at $z=0$, substituting these expressions into (3.2) and choosing the $c, C$, etc. to satisfy (3.1). This then yields 


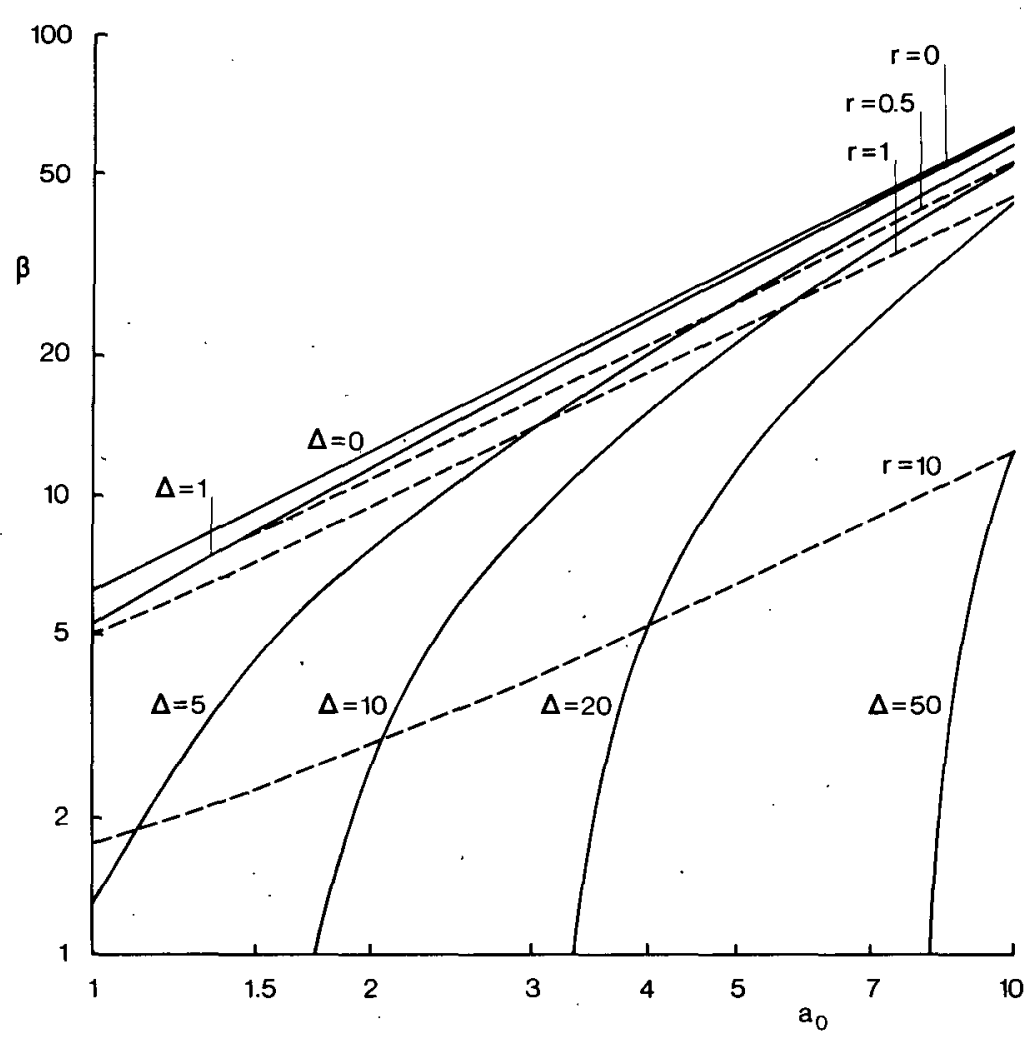

FIG. 5. Curves of constant $r$ (dashed) and $\Delta$ (solid) in the parametric domain ( $\beta$, $\left.a_{0}\right) . \Delta$ is the supercriticality $(6.2)$, and $r$ is the ratio of the maximum eddy potential enstrophy predicted by weakly-nonlinear theory, $(6.5)$, to the rigorous upper bound (4.32). The curves $\Delta=0$ and $r=0$ exactly coincide.

a bound on either the eddy potential enstrophy or the eddy APE at $z=0$.

While this procedure gives a rigorous saturation bound for any choice of a stable basic state, the interest is of course in deriving the best (i.e. tightest) such bound. For a general profile $\hat{U}(y, z)$, the optimization problem associated with the consideration of all possible basic states $(\Psi, Q)$ is evidently rather complicated, and could only be treated numerically. However, $P$. $H$. Haynes has shown by explicit construction that any particular saturation bound may be improved upon (though possibly only slightly) if the basic-state potential-vorticity gradient $Q_{y}$ varies smoothly in the neighbourhood of a point where it takes an extremum (Shepherd 1988b, appendix C). This means that one is justified in restricting attention to functions $Q_{y}$ that take their extrema over finite intervals, which is fortunate insofar as it is only for such basic states that the optimization problem is, in general, analytically tractable. (The difficulty is that the parameters $c, C$, etc. are functionals of $(\Psi, Q)$, and are therefore global rather than local properties of the basic state.) Moreover, the calculations of section 4 for the Charney problem suggest that one in fact does best by restricting attention to basic states with $Q_{y}$ constant throughout the domain; the point is that although one could reduce $\bar{q}(0)$ in the interior by choosing a basic state $U(y, z)$ that matched onto the initial profile $\hat{U}(y, z)$ at some $z$ (provided the flow was stable above this), the associated reduction of $\iint e^{-z / h} \bar{q}^{2}(0) d y d z$ is so small (because of the density factor) that it is more than offset by the fact that $C / c$ must then exceed unity.

For simplicity of presentation, we make the assumption of an isothermal semi-infinite atmosphere, with (4.2) and a constant scale height $H$; however, the extension to more general stratifications would be a straightforward exercise. Choosing $\xi$ to be the maximum vertical shear $\partial \hat{U} / \partial z$ (presumed positive), one may then employ the nondimensionalization of section 4. In these dimensionless units, it is convenient to write the potential vorticity of the initial zonal-mean state as

$$
\bar{P}(y, z, t=0) \equiv \zeta(y, z ; \lambda)+f+\beta y,
$$

and the surface temperature distribution as (proportional to)

$$
\bar{\Phi}_{z}(y, z=0, t=0) \equiv-W(y ; \lambda)
$$


the meridional gradient of (7.3) is of course just

$$
\begin{aligned}
\vec{\Phi}_{z y}(y, z & =0, t=0) \\
& =-\frac{\partial \hat{U}}{\partial z}(y, z=0, t=0)=-w(y),
\end{aligned}
$$

with $w \equiv d W / d y$. By hypothesis, $w(y) \leqslant 1$. The parameter $\lambda$ in (7.2) and (7.3) denotes the $(y-)$ constant of integration associated with the streamfunction $\bar{\Phi}$. For the moment, it is left free.

As suggested in the above discussion, we consider stable basic states of the form (4.27), with $Q_{y}$ constant throughout the domain and $\Psi_{z y}$ constant at $z=0$, namely

$$
C=c=\beta-h^{-1} u_{1}, \quad C_{1}=c_{1}=u_{1} .
$$

It then follows from (4.8), (4.10) (with $u_{2}=0$ and $b$ $=\infty)$, and (7.2), (7.3) that the initial disturbance (7.1) may be written as

$$
\begin{aligned}
q(t & =0)=\bar{P}(t=0)-Q+P^{\prime}(t=0) \\
& =\zeta(y, z ; \lambda)+h^{-1} u_{1}(y-\lambda)+P^{\prime}(t=0)
\end{aligned}
$$

in the interior, and

$$
\begin{aligned}
& \psi_{z}(t=0)=\bar{\Phi}_{z}(t=0)-\Psi_{z}+\Phi_{z}^{\prime}(t=0) \\
&=-W(y ; \lambda)-u_{1}(y-\lambda)+\Phi_{z}^{\prime}(t=0)
\end{aligned}
$$

at $z=0$.

\section{a. A bound on the eddy potential enstrophy}

The eddy potential enstrophy is bounded according to (4.17a); using (7.5), (7.6) and (7.7), and noting the orthogonality of zonal-mean and eddy components, (4.17a) takes the form

$$
\begin{array}{r}
\frac{1}{2} \iint e^{-z / h} \overline{P^{\prime 2}}(t) d y d z \leqslant \frac{1}{2} \iint e^{-z / h}\{\zeta(y, z ; \lambda) \\
\left.+h^{-1} u_{1}(y-\lambda)\right\}^{2} d y d z+Z_{0}+\frac{\beta-h^{-1} u_{1}}{u_{1}} \\
\times\left\{\frac{1}{2} \int\left\{W(y ; \lambda)+u_{1}(y-\lambda)\right\}^{2} d y+Y_{0}\right\} \\
=\alpha_{1} u_{1}^{-1}+\alpha_{2}+\alpha_{3} u_{1},
\end{array}
$$

where

$$
\begin{gathered}
\alpha_{1} \equiv \frac{\beta}{2} \int W^{2}(y ; \lambda) d y+\beta Y_{0}, \\
\alpha_{2} \equiv \beta \int W(y ; \lambda)(y-\lambda) d y-\frac{1}{2 h} \int W^{2}(y ; \lambda) d y \\
+\frac{1}{2} \iint e^{-z / h} \zeta^{2}(y, z ; \lambda) d y d z+Z_{0}-h^{-1} Y_{0}, \\
\alpha_{3} \equiv h^{-1} \iint e^{-z / h} \zeta(y, z ; \lambda)(y-\lambda) d y d z \\
+\frac{\beta}{2} \int(y-\lambda)^{2} d y-h^{-1} \int W(y ; \lambda)(y-\lambda) d y .
\end{gathered}
$$

For the purpose of this discussion we presume that the initial eddy amplitude is not too large: specifically that

$$
Y_{0} \leqslant O(1), \quad Z_{0} \leqslant O\left(h^{-1}\right)
$$

Now, (7.8) evidently takes a local minimum at $u_{1}$ $=\left(\alpha_{1} / \alpha_{3}\right)^{1 / 2}$ whenever $\alpha_{3}>0\left(\alpha_{1}\right.$ is always positive $)$; however, to be an acceptable solution this local minimum must fall with the permissible range of $u_{1}$, which is

$$
0<u_{1}<\beta h .
$$

Consider first the regime $\beta h \gg 1$. In this case, $\alpha_{3}$ asymptotes to the value $\frac{\beta}{2} \int(y-\lambda)^{2} d y$, and

$$
\frac{\alpha_{1}}{\alpha_{3}} \approx \frac{\int W^{2}(y ; \lambda) d y+2 Y_{0}}{\int(y-\lambda)^{2} d y}
$$

which is positive and of order unity and therefore satisfies ( 7.10$)$. The bound on the eddy potential enstrophy is then given by $(7.8)$ with $u_{1}=\left(\alpha_{1} / \alpha_{3}\right)^{1 / 2}$, namely

$$
\begin{aligned}
& \alpha_{2}+2\left(\alpha_{1} \alpha_{3}\right)^{1 / 2} \approx \beta\left\{\int W(y ; \lambda)(y-\lambda) d y\right. \\
& \left.\quad+\left\{\left[\int(y-\lambda)^{2} d y\right]\left[\int W^{2}(y ; \lambda) d y+2 Y_{0}\right]\right\}^{1 / 2}\right\} .
\end{aligned}
$$

(Because $\zeta$ is generally $\mathrm{O}\left(h^{-1}\right)$, all but the first term in the expression for $\alpha_{2}$ is no greater than $O\left(h^{-1}\right)$ and therefore negligible in this regime.) Note that the expression on the right-hand side of $(7.12)$ is positive, by virtue of Schwarz's inequality $\left(x^{2} y^{2}\right)^{1 / 2} \geqslant \overline{x y}$. Moreover, (7.12) is $O(\beta)$, and therefore much smaller than $Z$ as defined by (4.34), which is $O\left(\beta^{2} h\right)$. To obtain the best bound, it is now simply a matter of choosing $\lambda$ so as to minimize (7.12). If the zonal-wind shear $w(y)$ is symmetric about the center of the channel, then the choice $\lambda=1 / 2$ will give the best bound. For the special case $W(y ; \lambda)=y-\lambda$ corresponding to the Charney problem, $(7.12)$ reduces to $\beta(\eta+1) / 12$ for $\lambda=1 / 2$, in agreement with (4.31) [ since $Z_{0}-h^{-1} Y_{0} \ll$ $\beta$ under the hypothesis (7.9)]; this in fact represents the extreme case, as other symmetric $w(y)$ must give smaller bounds. For more general functions $w(y)$, the minimum of (7.12) would have to be worked out explicitly for the case in question.

In the regime $\beta h \ll 1$, on the other hand, there is a problem insofar as $\alpha_{3}$ may be negative; moreover, even if it is positive, $\alpha_{1} / \alpha_{3}=\mathrm{O}(\beta h)$ so $u_{1}=\mathrm{O}\left[(\beta h)^{1 / 2}\right]$ $\gg \beta h$, violating (7.10). Because (7.8) diverges as $u_{1} \rightarrow 0$, this means that the minimum of $(7.8)$ compatible with $(7.10)$ is achieved in the limit $u_{1} \rightarrow \beta h$, and is given by 
$\frac{1}{2} \iint e^{-z / h}[\zeta(y, z ; \lambda)+\beta(y-\lambda)]^{2} d y d z+Z_{0}$.

This expression is identical to $Z$ as defined by (4.34), with $\mu=f+\beta \lambda$; hence the minima of (4.34) and of (7.13) must be the same. Therefore, as in the Charney problem, for the case $\beta h \ll 1$ one can apparently do no better than the global invariant $Z$ for a bound on the eddy potential enstrophy.

When $\beta h=O(1)$, it is difficult to make a general statement. If there are choices of $\lambda$ for which $\alpha_{3}>0$ and $\left(\alpha_{1} / \alpha_{3}\right)^{1 / 2}<\beta h$, then (7.8) has a local minimum (in $u_{1}$ ) which is acceptable, namely $\alpha_{2}+2\left(\alpha_{1} \alpha_{3}\right)^{1 / 2}$; and one may choose $\lambda$ (consistent with these conditions) to minimize this quantity. Since it is a local minimum of (7.8), it is guaranteed to be smaller than the minimum value of $Z$, though not by much since both quantities are $O(\beta)$. If, however, one cannot find a local minimum (in $u_{1}$ ) for any $\lambda$, then the best bound is found for $u_{1} \rightarrow \beta h$ and is simply (7.13), equivalently $Z$.

\section{b. $A$ bound on the eddy $A P E$ at $z=0$}

The eddy APE at $z=0$ is bounded according to (4.38), which for (7.5), (7.6) and (7.7) becomes

$$
\begin{gathered}
\left.\frac{1}{2} \int \overline{\Phi_{z}^{\prime 2}}(t) d y\right|_{z=0} \leqslant \frac{u_{1}}{\beta-h^{-1} u_{1}}\left\{\frac{1}{2} \iint e^{-z / h}\right. \\
\left.\times\left[\zeta(y, z ; \lambda)+h^{-1} u_{1}(y-\lambda)\right]^{2} d y d z+Z_{0}\right\} \\
+\frac{1}{2} \int\left\{W(y ; \lambda)+u_{1}(y-\lambda)\right\}^{2} d y+Y_{0} \\
=\frac{\alpha_{1}+\alpha_{2} u_{1}+\alpha_{3} u_{1}^{2}}{\beta-h^{-1} u_{1}} .
\end{gathered}
$$

It is straightforward to check that (7.14) takes extrema at

$$
u_{1}=\beta h \pm\left[\beta^{2} h^{2}+\alpha_{3}^{-1}\left(\beta h \alpha_{2}+\alpha_{1}\right)\right]^{1 / 2}
$$

whenever the discriminant in (7.15) is positive. Now, if $\alpha_{3}$ and $\beta h \alpha_{2}+\alpha_{1}$ are both positive (recall that $\alpha_{1}$ is necessarily positive) then both solutions ( 7.15 ) violate (7.10), and the best bound on the eddy APE is found by taking $u_{1} \rightarrow 0$ in (7.14), namely

$$
\frac{i}{2} \int W^{2}(y ; \lambda) d y+Y_{0} \text {. }
$$

But (7.3) implies that this is just the total APE at $z$ $=0$, viz. the right-hand side of (4.41). This outcome was of course obtained in the Charney problem, for which $\alpha_{3}=\beta / 24$ and $\beta h \alpha_{2}+\alpha_{1}=\left(\beta^{2} h / 12\right)+(\beta /$ $24)+\beta h Z_{0}$ (taking $\lambda=1 / 2$ ). However, in the general case there is now a possibility of doing better than (7.16), provided that there are choices of $\lambda$ for which

$$
\alpha_{3}<0 \text { or } \beta h \alpha_{2}+\alpha_{1}<0 \text {, }
$$

but not both, and for which the discriminant in (7.15) is positive. In such cases, the minus root of (7.15) may be substituted into (7.14), and the resulting expression minimized over all such acceptable $\lambda$. However, it can be shown that this state of affairs cannot exist in the regime $\beta h \ll 1$, and that it can give only an asymptotically-negligible improvement in the regime $\beta h \gg 1$.

\section{Implications for transient-eddy parameterization}

The rigorous saturation bounds derived in this paper have a direct application in the context of transienteddy parameterization schemes for use in simple climate models. Traditionally, such schemes have usually been based on notions of eddy diffusivity, with a spatially varying diffusivity coefficient whose form is chosen according to linear instability theory (e.g., Green 1970 , et seq.) Such an approach is however difficult to justify, for reasons which need not be spelled out here. An alternative philosophy is suggested by the so-called "Transformed Eulerian Mean" formulation of the governing equations (Andrews and McIntyre 1976): there the effect of the eddies is collected in a single term, the divergence of the Eliassen-Palm flux $\nabla \cdot F$, which is itself related to eddy growth and decay. In the special case of weakly-dissipative flow, small-amplitude eddies, and no thermal driving, the zonal-mean' flow evolution is determined according to the system

$$
\begin{gathered}
\frac{\partial \bar{u}}{\partial t}-f_{0} \bar{v}^{*}=\nabla \cdot \mathbf{F}, \\
\frac{\partial \bar{\theta}}{\partial t}+\frac{d \Theta_{s}}{d z} \bar{w}^{*}=0 \\
\frac{\partial \bar{v}^{*}}{\partial y}+\frac{1}{\rho} \frac{\partial}{\partial z}\left(\rho \bar{w}^{*}\right)=0, \\
f_{0} \Theta_{s} \frac{\partial \bar{u}}{\partial z}=-g \frac{\partial \bar{\theta}}{\partial y}
\end{gathered}
$$

while the eddies themselves satisfy the conservation law

$$
\frac{\partial \hat{A}}{\partial t}+\nabla \cdot \mathbf{F}=0
$$

In the above $\hat{A}$ is the linearized Eliassen-Palm wave activity, just the negative of the zonal average of the right-hand side of (2.12), though here taking the zonalmean flow as the basic state, and (8.2) corresponds to the zonal average of (2.13). Thus, given knowledge of the evolution of $\hat{A}$, one can deduce $\nabla \cdot \mathbf{F}$; and from $\nabla \cdot F$, one may infer the zonal-mean atmospheric response [which is a combination of mean-flow "acceleration" $\left(\bar{u}_{t}, \bar{\theta}_{t}\right)$ and "residual circulation" $\left(\bar{v}^{*}, \bar{w}^{*}\right)$ in the meridional plane, together with a surface-pressure tendency (Haynes and Shepherd 1989)]. For a complete description of the problem, the surface con- 
tribution to the wave activity must be included. It may either be given by a separate term [just minus the smallamplitude form of $B(\theta, \theta)$ given by (2.15)], or alternatively incorporated as a surface contribution to $\hat{A}$ (Bretherton 1966; Hoskins et al. 1985). For the purpose of this discussion the latter choice is followed.

We now focus attention on the specific problem of parameterizing transient-eddy fluxes due to synopticscale baroclinic instability, and consider how the saturation bounds derived here may be used for this purpose. It must be emphasized that the nature of this discussion is purely heuristic and very speculative, and intended only to point the way towards a full quantitative description. Thus the arguments will be far from rigorous, and in particular will involve a judicious mixture of both linear and nonlinear ideas.

The results of numerically simulated nonlinear "life cycles" of baroclinic waves (Simmons and Hoskins 1978; Edmon et al. 1980) suggest the following sequence of events ( see Hoskins 1983; Held and Hoskins 1985): (i) linear growth near the surface, much as in the Charney (1947) model; (ii) nonlinear saturation, with an eddy APE cascade to small scales on the surface (frontogenesis) leading to dissipation; (iii) Rossbywave propagation from near-surface levels to the tropopause, and generally also equatorward; (iv) Rossbywave "breaking" and an associated enstrophy cascade to small scales in the vicinity of the subtropical jet. In terms of the E-P wave activity $\hat{A}$, stage (i) corresponds to growth of positive $\hat{A}$ in the near-surface interior region, and of negative $\hat{A}$ of the same magnitude concentrated at the lower surface (or negative $A$ and positive $B$, in the notation of section 2). The total wave activity, including the boundary contribution, must remain zero because it is zero initially (since the basic flow is here the initial zonal-mean flow), but this constraint does not apply to the eddy wave activity $\hat{A}$.

Associated with this vertical dipole structure in $\hat{A}$ is a vertical E-P flux F, or equivalently a poleward heat flux, as one expects from baroclinic instability. The magnitude of the net heat flux can then be determined on the basis of the saturation bounds on the surface eddy APE, and its vertical structure from the characteristics of the unstable Charney modes. This is in fact essentially no more than has already been proposed in the studies of Lindzen and Farrell (1980) and Branscome (1983) on heat-flux parameterization. Given the net heat flux (equivalently the vertical E-P flux), the effect on the mean flow can be fourd from (8.1) together with appropriate boundary conditions. Hayashi (1985, appendix D) shows the mean-flow changes associated with a growing Charney mode, though without allowing the concomitant surface-pressure tendency which would undoubtedly affect his result (see Haynes and Shepherd 1989).

The more difficult part of the problem, however, concerns the eddy momentum fluxes associated with stages (iii) and (iv). To address this aspect of the parameterization problem, we now propose to invoke the saturation bounds on the eddy potential enstrophy to determine how much eddy wave activity $\hat{A}$ is transported from the near-surface regions to the tropopause level. Because the unstable Charney-like modes in the life cycles are relatively shallow, we may idealize the problem by presuming that the regime $\gamma \ll 1$ (or $\beta h$ $\gg 1$ ) is the relevant one, in which case the meridional potential-vorticity gradient may be approximated by $\beta_{0}$. Then the net $\hat{A}$ in the interior, following saturation of the baroclinic instability, may be estimated as the eddy potential enstrophy divided by $\beta_{0}$, and is thus constrained by the saturation bounds. Note that it is not possible to estimate this $\hat{A}$ [relative to the zonalmean flow at the end of stage (ii)] from the surface part of $\hat{A}$ (relative to the initial zonal-mean flow) following saturation. This is because it is only the total (i.e. finite-amplitude) wave activities $A$ and $B$ that are linked, and these involve non-negligible zonal-mean disturbance components.

Then given the new $\hat{A}$ in the interior, it is presumed [according to stage (iii)] that it propagates (linearly or nonlinearly) vertically and meridionally. Where it goes will generally be a sensitive matter, determined in part by the "refractive index" associated with the relevant zonal-mean state (Hoskins, McIntyre and Thorncroft, manuscript in preparation). For the purpose of this discussion, however, we simply presume that it goes to some region near the tropopause where it is dissipated at small scales following an enstrophy cascade (cf. Held and Hoskins 1985). Then the magnitude of $\nabla \cdot F$ both at this region (where it is negative) and near the surface (where it is positive) is known from the saturation bounds, and the effects on the mean flow may be determined from (8.1). When the two regions of nonzero $\nabla \cdot \mathbf{F}$ are offset meridionally, as they generally will be, then the calculations of Pfeffer (1987) suggest that the net effect will be a meridional dipole in $\partial \bar{u} / \partial t$, reflecting eddy momentum flux convergence and divergence.

The total problem is thus closed by small-scale dissipation in two regions: near the subtropical jet, and at the surface itself. This phenomenological hypothesis is indeed borne out in the life-cycle experiments (see Held and Hoskins 1985, Fig. 4). The dissipation is of course essentially dynamical, in the sense that it is driven by nonlinear cascades (of potential enstrophy in the interior, and of APE at the surface) to small scales, which are associated with the large-scale flow itself; moreover, it acts to destroy both positive $\hat{A}$ (at the tropopause) and negative $\hat{A}$ (at the surface), as it must since there is no net production of $\hat{A}$ by the baroclinic instability process. However, it is the spatial separation of the two dissipation processes which is responsible for the net effects on the mean flow.

Perhaps needless to say, this picture is highly ideal- 
ized and will need to be tested quantitatively. In particular, surface damping will be expected to modify the behavior, although preliminary studies have shown that the effects on the life cycles are more quantitative than qualitative (M. Blackburn, personal communication 1987). Work along these lines is currently in progress and will be reported on in due course.

\section{Discussion}

This paper addresses the question of finite-amplitude saturation of baroclinic instability in a continuously stratified, quasi-geostrophic fluid. It thereby extends the analysis of Part I of this study (Shepherd 1988a), on the two-layer model, to a more geophysically relevant setting. By appealing to a nonlinear Liapunov (normed) stability theorem, (3.4), which is the finiteamplitude generalization of the well-known CharneyStern theorem for zonal flows, rigorous upper bounds have been derived both on the depth-integrated eddy potential enstrophy and on the eddy available potential energy at the ground. These bounds are unconstrained by any assumptions about the nature of the initial nonzonal disturbance, and thus are not tied in any way to the concept of normal-mode instability.

The character of the results depends on the dimensionless external parameter $\gamma=f_{0}{ }^{2} \xi / \beta_{0} N^{2} H$, where $\xi$ is the maximum vertical shear of the zonal wind, and the other symbols have their usual meaning (see sections 2 and 4). When $\gamma \gg 1$, corresponding to "deep" unstable modes (vertical scale $\approx H$ ), the bound on the eddy potential enstrophy, (7.13), is just the total potential enstrophy in the system; but when $\gamma \ll 1$, corresponding to "shallow" unstable modes (vertical scale $\approx \gamma H$ ), the eddy potential enstrophy is bounded, by (7.12), well below the total amount available in the system. In neither case can the bound on the eddy APE, (7.16), prevent a complete neutralization of the surface temperature gradient, which is in accord with numerical experience (Simmons and Hoskins 1978).

For the special case of the Charney (1947) model of baroclinic instability, the bounds on the eddy potential enstrophy and eddy APE are given in terms of dimensional variables by (4.36) and (4.43), respectively, and have the same character as the general results described above. In the important limit of infinitesimal initial eddy disturbance amplitude, the dimensionless bound on the eddy potential enstrophy is (4.32), namely $\beta$ / 6 for $\gamma \leqslant 1$ and (asymptotically) $\frac{1}{24 h}$ for $\gamma \gg 1$; here $\beta$ and $h$ are the dimensionless beta and scale height parameters (see section 4 ). These bounds are very similar to (though of course generally larger than) ad hoc estimates based on baroclinic-adjustment arguments (section 5 and Fig. 2).

The system of equations used here is the standard quasi-geostrophic set, which is appropriate for the problem under consideration. It is, however, a straightforward matter to show that the more general "modified quasi-geostrophic equations" (White 1977) also allow a pseudomomentum conservation lav and generalized Charney-Stern theorem, and that saturation bounds likewise obtain. The details are provided in appendix $A$.

The theory in this paper has been worked out under the assumption of conservative (inviscid, unforced) flow. It turns out, however, that the results go through for a certain kind of forced-dissipative problem, namely that where the potential vorticity and surface potential temperature are relaxed back to the initial unstable state. In place of (2.1) and (2.4), then, one considers

$$
\begin{gathered}
\frac{D P}{D t}=-\nu\left(P-P_{e}\right), \quad \text { for } z_{1} \leqslant z \leqslant z_{2}, \\
\frac{D}{D t} \Phi_{z}=-\nu\left(\Phi_{z}-\Phi_{e z}\right), \quad \text { at } z=z_{1}, z_{2} .
\end{gathered}
$$

Here $\nu$ is the relaxation coefficient and the subscript $e$ (for equilibrium) denotes the (constant) initial unstable zonal-mean state, viz. $P_{e}=\bar{P}(t=0)$; in the atmospheric context such a state might represent the radiative-convective equilibrium state, for example. The system (9.1) can also be thought of as one having dissipation proportional to the potential vorticity in the interior, and to the potential temperature at the surface, together with a constant forcing to ensure that the initial (unstable) zonal-mean state is a steady solution of the governing equations. Although the pseudomomentum conservation law (2.18) now has nonconservative terms on the right-hand side, so that $J(t) \neq J(0)$ generally, and although $d|J| / d t$ may in fact be positive, nevertheless one may establish that

$$
|J(t)| \leqslant|J(0)| \text {, }
$$

from which the stability theorem (3.4) follows directly; thus all the results derived in this paper continue to hold for the system (9.1).

The proof of (9.2) for the system (9.1) is given for the case of two-dimensional (barotropic) flow in Shepherd (1988b, §4.1), where $P=\Phi_{x x}+\Phi_{y y}+f_{0}+\beta_{0} y$ rather than (2.2). Since the proof does not depend on the detailed definition of $P$, but only on the form (9.1) together with the nature of the pseudomomentum invariant $A(Q, q)$, it is a straightforward exercise to verify that it goes through for the present case. (This is most easily seen by treating the surface contribution as part of the potential vorticity, and imposing the homogeneous lower boundary condition $\Phi_{z}=0$; see Bretherton 1966; Hoskins et al. 1985.)

Whatever the physical relevance of the forced-dissipative system (9.1), it does correspond to the kind of system often used in theoretical and modeling studies. It arises, for example, when dissipation is provided by Rayleigh friction and Newtonian cooling with equal, constant relaxation coefficients. On the other hand, it 
has not been possible to derive a result corresponding to (9.2) when these coefficients are unequal, or when Ekman damping is applied at the surface. The extent to which the saturation bounds can be salvaged in general kinds of forced-dissipative problems is an interesting subject for further study.

Although the saturation bounds derived here have been obtained by varying the vertical structure of the basic flow, the method is evidently not limited to this sort of approach. For example, the study of Gutowski (1985) suggests that changes in the static stability associated with vertical heat fluxes may be a crucial part of mean-flow adjustment to baroclinic instability. It would therefore be interesting to determine the saturation bounds obtainable by considering basic flows with different static stability profiles (in effect, taking part of the "reference state" $\theta_{s}(z)$ as the initial disturbance). Similarly, James (1987) has proposed that adjustment involves changes in the barotropic component of the mean flow, and one might try to see how much of James's "barotropic governor" could be captured through the saturation-bound approach.

One application of these bounds is in providing a constraint for evaluating the validity of approximate (e.g. weakly nonlinear or low-order) theories. For example, Pedlosky's (1979b) weakly nonlinear theory for the Charney problem was shown (section 6) to violate the rigorous saturation bounds for $O(1)$ supercriticality. Another application is that of providing an eddy-amplitude closure for transient-eddy parameterization schemes ( section 8), a subject of both theoretical and practical importance. In this regard, and indeed more generally, it would be of considerable interest to determine the extent to which the saturation bounds give quantitatively accurate estimates of maximum eddy amplitudes under various conditions.

Although this last question can only be properly addressed through fully nonlinear numerical simulations, it is nevertheless fairly clear that there is a parameter regime where the saturation bounds will give hopelessly large overestimates (I. M. Held, personal communication 1988); namely the homogeneous limit of a very broad jet. When the Rossby deformation radius $\mathrm{NH} /$ $f_{0}$ (which is an upper bound on the eddy length scale) is much less than the width of the jet, $\hat{L}$, then the homogeneous limit is reached where eddy statistics are independent of $\hat{L}$ (Haidvogel and Held 1980). If we take $\hat{L} \approx L$, the channel width (as in the Charney problem), then the homogeneous limit is the limit $h$ $\ll 1$, and the spatially-integrated eddy potential enstrophy and eddy APE would be expected to scale as $L$. The rigorous saturation bound (4.36), on the other hand, scales as $L^{3}$, and so will be a considerable overestimate as $L \rightarrow \infty$. This implies, inter alia, that the flow cannot be neutralized as in the adjustment arguments. The physical reasons for this involve the fact that the saturation bound must cover the (extreme) case of the gravest meridional mode, but the gravest mode is unstable in the homogeneous limit and proves to be irrelevant to the finite-amplitude dynamics (Haidvogel and Held 1980). The earth's atmosphere is far from the homogeneous limit, but these considerations might be relevant on Jupiter, for example.

Another limitation of this study is that the method used here would seem to be restricted to quasi-geostrophic dynamics. This is because the Liapunov stability theorem on which the method relies does not appear to exist in the fully ageostrophic case (Abarbanel et al. 1986), unless one can make a priori assumptions about the spatial structure of the disturbance. Of course, for practical purposes such assumptions (e.g. the existence of a minimum vertical scale) may be quite reasonable in certain circumstances.

Acknowledgments. This research has been supported by the UK Natural Environment Research Council and by St. Catharine's College, Cambridge.

\section{APPENDIX A}

\section{Generalization to the Modified Quasi-geostrophic Equations}

The system of equations used in the body of this paper is the standard quasi-geostrophic set, which is derived under the quasi-Boussinesq assumption $f_{0}^{2} L^{2} / g H \ll 1$. Although this assumption is reasonable for synoptic-scale motions of the kind under consideration here, it becomes highly suspect (if not invalid) for planetary-scale motions. When this parameter is taken to be of order unity, the potential vorticity equation (2.1) remains unchanged, but the boundary condition (2.4) becomes

$$
\frac{D}{D t}\left(\Phi_{z}-\frac{N^{2}}{g} \Phi\right)=0
$$

Similarly, while the expression for the potential vorticity is still (2.2), the potential temperature is given by

$$
\theta=\frac{\Theta_{s} f_{0}}{g}\left(\Psi_{z}-\frac{N^{2}}{g} \Psi\right), \quad \theta=\frac{\Theta_{s} f_{0}}{g}\left(\psi_{z}-\frac{N^{2}}{g} \psi\right),
$$

instead of (2.16). White (1977) refers to this as the modified quasi-geostrophic set of equations. The purpose of this appendix is simply to point out that the pseudomomentum conservation law and generalized Charney-Stern theorem derived in sections 2 and 3 go through for the modified equations without alteration, and that saturation bounds will therefore follow.

The crucial point about the changes implied by (A1) and (A2) is that the potential temperature at the boundaries is still a materially conserved quantity, viz. 


$$
\frac{D}{D t}(\Theta+\theta)=0 \quad \text { at } \quad z=z_{1}, z_{2}
$$

and so the function $B(\theta, \theta)$ defined by $(2.15)$ remains appropriate. The only difference is that $(2.17)$ becomes

$$
\frac{D B}{D t}=\rho\left(f_{0} / N\right)^{2} \psi_{x}\left(\psi_{z}-\frac{N^{2}}{g} \psi\right),
$$

but this has no effect on (2.18) since the new term is proportional to $\psi_{x} \psi$ and thus vanishes under a zonal average. The generalized Charney-Stern theorem (3.4) goes through accordingly. Blumen (1978) has shown that the pseudoenergy conservation law and associated Arnol'd stability theorem hold for the linearized version of the modified quasi-geostrophic equations.

With regard to the saturation bounds, the details of the calculations presented here (especially in sections 5 and 7) would generally require modification. However, because both the unstable Charney profile (4.1) and the stable basic flow (4.27) have zero velocity at the ground, the extra term in (A2) vanishes anyway and the saturation bounds derived in section 4 apply virtually as they stand. The only modification is that in their dimensionless versions, the expressions for the eddy APE (including that for $Y_{0}$ ) require the correct version of dimensionless potential temperature according to (A2).

\section{APPENDIX B}

\section{Check on the Expression (6.5)}

From Pedlosky's (1979b) equation (3.44), the leading-order correction to the zonal-mean potential vorticity is given by

$$
q_{0} e^{-\beta_{c} z}|A|^{2} \pi l \sin (2 \pi l y),
$$

so taking $\lambda=1 / 2, \mu=f+\beta / 2, l=1$, and $q_{0} \approx \beta_{c}$ as before, this yields

$$
\begin{aligned}
\bar{P}-\mu=\left(\beta+h^{-1}\right) & \left(y-\frac{1}{2}\right) \\
& +|\Delta| \beta_{c} e^{-\beta_{c} z}|A|^{2} \pi \sin (2 \pi y) .
\end{aligned}
$$

At $t=0$, the second term of the above expression is negligible (by hypothesis); when $|A|^{2}=|A|_{\max }^{2}$, the ratio of the two terms is $O\left(|\Delta|^{-1}|A|^{-2}\right)$ $=\mathrm{O}\left(|\Delta|^{-1} \beta_{c}{ }^{2}\right) \gg 1$ and the first term dominates. Therefore, using (4.33a), the maximum eddy potential enstrophy is given by

$$
\begin{aligned}
& \frac{1}{2} \iint e^{-z / h} \overline{P^{\prime 2}} d y d z \\
& =\left.\frac{1}{2} \iint e^{-z / h}(\bar{P}-\mu)^{2} d y d z\right|_{\left(|A|^{2}=0\right)} \\
& \quad-\left.\frac{1}{2} \iint e^{-z / h}(\bar{P}-\mu)^{2} d y d z\right|_{\left(|A|^{2}=|A|_{\text {max }}^{2}\right)}
\end{aligned}
$$

$$
\begin{aligned}
\approx & -|\Delta| \beta_{c}|A|_{\max }^{2} \pi \iint e^{-z / h}\left(\beta+h^{-1}\right) \\
& \times\left(y-\frac{1}{2}\right) e^{-\beta_{c} z} \sin (2 \pi y) d y d z \approx \frac{1}{2}|\Delta| \beta|A|_{\max }^{2},
\end{aligned}
$$

which agrees with (6.5) to leading order in $|\Delta|^{1 / 2}$.

\section{REFERENCES}

Abarbanel, H. D. I., D. D. Holm, J. E. Marsden and T. Ratiu, 1986: Nonlinear stability analysis of stratified fluid equilibria. Phil. Trans. Roy. Soc. London, A318, 349-409.

Andrews, D. G., and M. E. McIntyre, 1976: Planetary waves in horizontal and vertical shear: The generalized Eliassen-Palm relation and the mean zonal acceleration. J. Atmos. Sci., 33, 2031-2048.

Arnol'd, V. I., 1966: On an a priori estimate in the theory of hydrodynamical stability. Izv. Vyssh. Uchebn. Zaved. Matematika, 54(5), 3-5. [English transl.: Amer. Math. Soc. Transl. Series 2, 79, 267-269 (1969).]

Blumen, W., 1978: A note on horizontal boundary conditions and stability of quasi-geostrophic flow. J. Atmos. Sci., 35, 13141318.

Branscome, L. E., 1983: A parameterization of transient eddy heat flux on a beta-plane. J. Atmos. Sci., 40, 2508-2521.

Bretherton, F. P., 1966: Critical layer instability in baroclinic flows. Quart. J. Roy. Meteor. Soc., 92, 325-334.

Charney, J. G., 1947: The dynamics of long waves in a baroclinic westerly current. $J$. Meteorol., 4, 135-162.

Eady, E. T., 1949: Long waves and cyclone waves. Tellus, 1, 33-52.

Edmon, H. J., B. J. Hoskins and M. E. McIntyre, 1980: EliassenPalm cross-sections for the troposphere. J. Atmos. Sci., 37, 26002616. (Corrigenda, 38, 1115.)

Green, J. S. A., 1960: A problem in baroclinic instability. Quart. $J$. Roy. Meteor. Soc., 86, 237-251.

- 1970: Transfer properties of the large-scale eddies, and the general circulation of the atmosphere. Quart. J. Roy. Meteor. Soc., 96, 157-184.

Gutowski, W. J., 1985: Baroclinic adjustment and midlatitude temperature profiles. J. Atmos. Sci., 42, 1733-1745.

Haidvogel, D. B., and I. M. Held, 1980: Homogeneous quasi-geostrophic turbulence driven by a uniform temperature gradient. J. Atmos. Sci., 37, 2644-2660.

Hayashi, Y., 1985: Theoretical interpretations of the Eliassen-Palm diagnostics of wave-mean flow interaction. Part I: Effects of the lower boundary. J. Meteor. Soc. Jpn., 63, 497-512.

Haynes, P. H., and T. G. Shepherd, 1989: The importance of surfacepressure changes in the response of the atmosphere to zonallysymmetric thermal and mechanical forcing. Quart. J. Roy. Meteor. Soc., in press.

Held, I. M., 1978: The vertical scale of an unstable baroclinic wave and its importance for eddy heat flux parameterizations. $J$. Atmos. Sci., 35, 572-576.

- 1985: Pseudomomentum and the orthogonality of modes in shear flows. J. Atmos. Sci., 42, 2280-2288.

circulation of the troposphere. Advances in Geophysics, 28A, 3-31, Academic Press.

Holm, D. D., J. E. Marsden, T. Ratiu and A. Weinstein, 1985: Nonlinear stability of fluid and plasma equilibria. Phys. Rep., 123, $-1-116$.

Hoskins, B. J., 1983: Modelling of the transient eddies and their feedback on the mean flow. Large-Scale Dynamical Processes in the Atmosphere, B. J. Hoskins and R. P. Pearce, Eds., Academic Press, 169-199.

- M. E. McIntyre and A. W. Robertson, 1985: On the use and significance of isentropic potential vorticity maps. Quart. J. Roy. Meteor. Soc., 111, 877-946. 
James, I. N., 1987: Suppression of baroclinic instability in horizontally sheared flows. J. Atmos. Sci., 44, 3710-3720.

Killworth, P. D., and M. E. McIntyre, 1985: Do Rossby-wave critical layers absorb, reflect or over-reflect? J. Fluid Mech., 161, 449492.

Lindzen, R. S., and B. Farrell, 1980: The role of polar regions in global climate, and a new parameterization of global heat transport. Mon. Wea. Rev., 108, 2064-2079.

McIntyre, M. E., and T. G. Shepherd, 1987: An exact local conservation theorem for finite-amplitude disturbances to non-parallel shear flows, with remarks on Hamiltonian structure and on Arnol'd's stability theorems. J. Fluid Mech., 181, 527-565.

Pedlosky, J., 1970: Finite-amplitude baroclinic waves. J. Atmos. Sci., $27,15-30$

—, 1979a: Geophysical Fluid Dynamics. Springer-Verlag, 624 pp. , 1979b: Finite-amplitude baroclinic waves in a continuous model of the atmosphere. J. Atmos. Sci., 36, 1908-1917.

Pfeffer, R. L., 1987: Comparison of conventional and transformed Eulerian diagnostics in the troposphere. Quart. J. Roy. Meteor. Soc., 113, 237-254.

Phillips, N. A., 1954: Energy transformations and meridional circulations associated with simple baroclinic waves in a two-level quasi-geostrophic model. Tellus, 6, 273-286.

Salmon, R., 1980: Baroclinic instability and geostrophic turbulence. Geophys. Astrophys. Fluid Dyn., 15, 167-211.
Schoeberl, M. R., and R. S. Lindzen, 1984: A numerical simulation of barotropic instability. Part I: Wave-mean flow interaction. J. Atmos. Sci., 41, 1368-1379.

Shepherd, T. G., 1988a: Nonlinear saturation of baroclinic instability. Part I: The two-layer model. J. Atmos. Sci., 45, 2014-2025.

- 1988b: Rigorous bounds on the nonlinear saturation of instabilities to parallel shear flows. J. Fluid Mech., 196, 291-322.

Simmons, A. J., and B. J. Hoskins, 1976: Baroclinic instability on the sphere: Normal modes of the primitive and quasi-geostrophic equations. J. Atmos. Sci., 33, 1454-1477.

$\longrightarrow$, and $-1978:$ The life cycles of some nonlinear baroclinic waves. J. Atmos. Sci., 35, 414-432.

Stone, P. H., 1978: Baroclinic adjustment. J. Atmos. Sci., 35, 561571.

Vallis, G. K., 1988: Numerical studies of eddy transport properties in eddy resolving and parametrized models. Quart. J. Roy. Meteor. Soc., 114, 183-204.

White, A. A., 1977: Modified quasi-geostrophic equations using geometric height as vertical coordinate. Quart. J. Roy. Meteor. Soc., 103, 383-396.

- , and A. M. Gadian, 1979: Baroclinic instability governed by the modified quasi-geostrophic equations. Quart. J. Roy. Meteor. Soc., 105, 759-766. 